ESAIM: COCV 18 (2012) 318-342

DOI: $10.1051 / \mathrm{cocv} / 2011004$
ESAIM: Control, Optimisation and Calculus of Variations

www.esaim-cocv.org

\title{
THE BACK AND FORTH NUDGING ALGORITHM FOR DATA ASSIMILATION PROBLEMS: THEORETICAL RESULTS ON TRANSPORT EQUATIONS
}

\author{
Didier Auroux ${ }^{1,2}$ ANd MaËLle NodeT ${ }^{2,3}$
}

\begin{abstract}
In this paper, we consider the back and forth nudging algorithm that has been introduced for data assimilation purposes. It consists of iteratively and alternately solving forward and backward in time the model equation, with a feedback term to the observations. We consider the case of 1-dimensional transport equations, either viscous or inviscid, linear or not (Burgers' equation). Our aim is to prove some theoretical results on the convergence, and convergence properties, of this algorithm. We show that for non viscous equations (both linear transport and Burgers), the convergence of the algorithm holds under observability conditions. Convergence can also be proven for viscous linear transport equations under some strong hypothesis, but not for viscous Burgers' equation. Moreover, the convergence rate is always exponential in time. We also notice that the forward and backward system of equations is well posed when no nudging term is considered.
\end{abstract}

Mathematics Subject Classification. 35Q35, 35R30, 65M32.

Received March 14, 2010. Revised September 20, 2010.

Published online January 19, 2011.

\section{INTRODUCTION}

Data assimilation is the set of techniques aiming to combine in an optimal way the mathematical information provided by the model equations and the physical information given by observations, in order to retrieve the state of a system. Several types of methods have been widely studied in the past decades. We can cite here interpolation, variational and stochastic methods. The first ones interpolate the measurements from the points of observation towards the grid points, the interpolation being weighted by the statistics of the observations [12]. Variational methods are based on the optimal control theory, and data assimilation is set as being a problem of constrained optimization. The goal is to minimize a cost function measuring the difference between the observations and the corresponding quantities provided by a model integration. The initial condition of the system can then be seen as a control vector [14]. Finally, the basic idea of stochastic methods is to consider the fields as the realization of a stochastic process and carry out Kalman filtering methods [7,11]. We can also mention one of the very first data assimilation schemes: the nudging method. Also known as Newtonian relaxation or dynamic initialization, it consists of adding a feedback term to the observations directly in the model equations [10].

Keywords and phrases. Data assimilation, inverse problems, linear transport equations, Burgers' equation.

${ }^{1}$ Laboratoire Dieudonné, Université de Nice Sophia Antipolis, Parc Valrose, 06108 Nice Cedex 2, France. auroux@unice.fr

2 INRIA, Grenoble, France.

${ }^{3}$ Université de Grenoble, Laboratoire Jean Kuntzmann, UMR 5224, Grenoble, France. maelle.nodet@inria.fr 
All these methods require extensive work, either from the implementation or the computation point of view. For instance, variational methods require the linearization of all operators and also the implementation of the adjoint model. They also need efficient optimization schemes, as the minimization is performed on spaces of huge dimension. On the other side, stochastic methods are somewhat easier to implement, but they require knowledge, storage and manipulations of huge matrices.

The Back and Forth Nudging (BFN) algorithm has recently been introduced as a simple and efficient method for solving data assimilation problems [1]. In most geophysical applications, data assimilation consists of estimating a trajectory, solution of a partial differential equation (PDE), from the knowledge of observations. These observations are usually sparse in time and space, and incorrect in the sense that they are not the restriction of a solution of the PDE model. One step of the BFN algorithm consists of solving first the model equation, in which a feedback to the observation solution is added, and then the same equation but backwards in time, with also a feedback term to the observations. Such forward and backward integrations provide a new value of the solution at the initial time $t=0$ and the aim of the BFN is to improve the quality of the initial condition.

The idea of the back and forth nudging is to use the difference between the observations and the model trajectory as a feedback control of the equations, both in the forward and backward integrations. This makes the numerical scheme extremely easy to implement, in comparison with both variational and stochastic methods, as we usually only consider diagonal (or even scalar) gain matrices. The back and forth nudging scheme can also be seen as an intermediate scheme between variational and stochastic methods, as the standard nudging technique has both variational (minimization of a compromise between the observations and the energy of the system) and stochastic (sub-optimal Kalman filter) interpretations [2].

In a linear case, the forward nudging method is nothing else than the Luenberger observer, also called asymptotic observer. In more general situations, past efforts in the theory of observers for systems described by PDEs include infinite dimensional observers for wave type equations and reversible systems [8,9], parabolic equations [19], viscous Burgers and shallow water equations [3,13]. Filter and observer design inspired by robust control feedback has been recently developed and studied in a standard (forward) way for medical imaging [16]. The BFN has also been extended to fit into the observers framework and has been applied to reversible systems in [17]. The aim of our paper is to provide a general study of the Back and Forth Nudging algorithm for geophysical type PDEs within the data assimilation framework. The goal is to recover the full state of the system given some observations distributed over space and time.

As a first approximation, we consider in this paper that the observations are correct (i.e. no observation error), and hence the observations satisfy the model equation. We consider various observation domains: first we assume that the observations $u_{\mathrm{obs}}(t, x)$ are available for any point $x$ and time $t$, second we assume that they are available for $t \in\left[t_{1}, t_{2}\right]$ and for all $x$, and third we consider that they are available for all $t$ over a given space domain. This is done through the time and space dependency of the feedback (or nudging) gain matrix $K(t, x)$ that is equal to 0 when the observations are not available.

Many numerical experiments in almost realistic situations suggest that this algorithm works well, and that the identified solution gets closer to the observations [2]. The goal of this paper is to prove some theoretical results and convergence properties in the particular case of transport equations, either viscous or inviscid, either linear or non-linear (Burgers' equation).

In Section 2, we consider one step of the BFN algorithm applied to a linear viscous transport equation.

In Section 3, we consider one step of the BFN algorithm applied to the viscous Burgers' equation.

Section 4 considers the extension of Theorem 2.4 to the inviscid case, for both linear transport and Burgers' equations. We first consider the linear case. An explicit counter-example for this case is detailed in Appendix 6.

We finally consider non viscous Burgers' equation, with periodic boundary conditions, and for a time $T$ such that there is no shock in the interval $[0, T]$.

Finally, some conclusions are given in Section 5. 


\section{Linear transport EQUATION WITH A VISCOUS TERM}

\subsection{Back and Forth Nudging algorithm}

From $[1,2]$ we recall the purpose and design of the Back and Forth Nudging algorithm, applied to the following partial differential equation:

$$
\left\{\begin{array}{r}
\partial_{t} u-\nu \partial_{x x} u+a(x) \partial_{x} u=0 \\
\left.u\right|_{x=0}=\left.u\right|_{x=1}=0
\end{array}\right.
$$

where

- the time period considered is $t \in[0, T]$;

- the space domain $\Omega$ is either the interval $[0,1]$ or the torus $\mathbb{R} / \mathbb{Z}$;

- $a(x) \in W^{1, \infty}(\Omega)$;

- $\nu>0$ is a constant.

The aim of the BFN algorithm is to recover the state $u$, solution of equation (2.1), given some observations $u_{\mathrm{obs}}$ on some subset $\omega$ of $[0, T] \times \Omega$ :

$$
u(t, x)=u_{\mathrm{obs}}(t, x), \quad(t, x) \in \omega \subset[0, T] \times \Omega,
$$

where $u_{\text {obs }}$ is obtained from an exact (but unknown) solution $u_{\text {true }}$ of equation (2.1), associated to an unknown initial condition $\left.u_{\text {true }}\right|_{t=0} \in L^{2}(\Omega)$ :

$$
u_{\mathrm{obs}}(t, x)=u_{\text {true }}(t, x),(t, x) \in \omega ; \quad u_{\mathrm{obs}}(t, x)=0,(t, x) \notin \omega
$$

The BFN algorithm consists in performing several iterations of the forward and backward nudging algorithms, as follows:

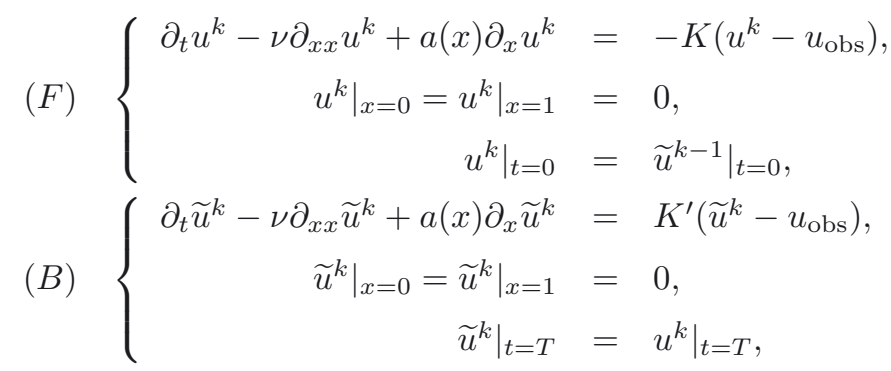

for $k \geq 0$, where

- $\left.\widetilde{u}^{-1}\right|_{t=0}$ is given;

- $K \in L^{\infty}([0 ; T] \times \Omega)$ and $K^{\prime} \in L^{\infty}([0 ; T] \times \Omega)$ are positive and their support in $(t, x)$ is exactly $\omega$, so that $K=K^{\prime}=0$ when and where observations are not available.

The aim of the BFN is to obtain the convergence of $u^{k}$ to $u_{\text {true }}$ for all $t$ and $x$, when $k$ tends to infinity. In the sequel, the observation domain (in space and time) will be given by the support of $K$ :

$$
\omega=\operatorname{Support}(K) \subset[0, T] \times \Omega \text {. }
$$




\subsection{Main result and comments}

We consider here one step of the BFN algorithm applied to a linear viscous transport equation:

$$
\begin{aligned}
& (F)\left\{\begin{aligned}
\partial_{t} u-\nu \partial_{x x} u+a(x) \partial_{x} u & =-K\left(u-u_{\mathrm{obs}}\right), \\
\left.u\right|_{x=0}=\left.u\right|_{x=1} & =0, \\
\left.u\right|_{t=0} & =u_{0},
\end{aligned}\right. \\
& (B)\left\{\begin{aligned}
\partial_{t} \widetilde{u}-\nu \partial_{x x} \widetilde{u}+a(x) \partial_{x} \widetilde{u} & =K^{\prime}\left(\widetilde{u}-u_{\text {obs }}\right), \\
\left.\widetilde{u}\right|_{x=0}=\left.\widetilde{u}\right|_{x=1} & =0 \\
\left.\widetilde{u}\right|_{t=T} & =u(T),
\end{aligned}\right.
\end{aligned}
$$

where $u_{\text {obs }}$ is obtained from $u_{\text {true }}$ according to $(2.2,2.3)$, where $u_{\text {true }}$ satisfies:

$$
\left\{\begin{aligned}
\partial_{t} u_{\text {true }}-\nu \partial_{x x} u_{\text {true }}+a(x) \partial_{x} u_{\text {true }} & =0 \\
\left.u_{\text {true }}\right|_{x=0}=\left.u_{\text {true }}\right|_{x=1} & =0 \\
\left.u_{\text {true }}\right|_{t=0} & =u_{\text {true }}^{0}
\end{aligned}\right.
$$

The following notations and hypotheses hold for all further cases:

\section{Notations 2.1.}

- The time period considered is $t \in[0, T]$;

- the space domain $\Omega$ is either the interval $[0,1]$ or the torus $\mathbb{R} / \mathbb{Z}$;

- the first equation $(F)$ is called the forward equation, the second one $(B)$ is called the backward one.

\section{Hypotheses 2.2.}

- $K \in L^{\infty}([0 ; T] \times \Omega)$ and $K^{\prime} \in L^{\infty}([0 ; T] \times \Omega)$ are positive and may depend on $t$ and $x$, but for the sake of simplicity, we will always assume that there exists a constant $\kappa \in \mathbb{R}_{+}^{*}$ such that $K^{\prime}(t, x)=\kappa K(t, x)$, and that Support $(K)=$ Support $\left(K^{\prime}\right)=\omega \subset[0, T] \times \Omega$;

- observations $u_{\mathrm{obs}}$ are available only on $\omega$;

- $a(x) \in W^{1, \infty}(\Omega)$

- $\nu>0$ is a constant;

- $u_{\text {true }}^{0} \in L^{2}(\Omega)$ and $u_{0} \in L^{2}(\Omega)$.

Using the previous notations, we recall the following straightforward result:

Proposition 2.3. Under Hypotheses 2.2, equation (2.5) has a unique solution $u_{\text {true }} \in C^{0}\left(0, T ; L^{2}(\Omega)\right) \cap$ $L^{2}\left(0, T ; H^{1}(\Omega)\right)$.

Then the following result holds true:

Theorem 2.4. Under Hypotheses 2.2, let $u_{\text {true }}$ be the solution of (2.5) given by Proposition 2.3 , and $u_{\mathrm{obs}}$ given by $(2.2,2.3)$. Then equation $(2.4-F)$ has a unique solution $u \in C^{0}\left(0, T ; L^{2}(\Omega)\right) \cap L^{2}\left(0, T ; H^{1}(\Omega)\right)$. Moreover:

(1) If $K(t, x)=K \in \mathbb{R}$, then equation $(2.4-B)$ has a unique solution $\tilde{u} \in C^{0}\left(0, T ; L^{2}(\Omega)\right) \cap L^{2}\left(0, T ; H^{1}(\Omega)\right)$ and thus one step of the BFN algorithm (2.4) is well posed. Moreover, if we denote

$$
\begin{aligned}
& w(t)=u(t)-u_{\text {true }}(t), \\
& \widetilde{w}(t)=\widetilde{u}(t)-u_{\text {true }}(t),
\end{aligned}
$$

then for all $t \in[0, T]$ :

$$
\widetilde{w}(t)=\mathrm{e}^{\left(-K-K^{\prime}\right)(T-t)} w(t)
$$

(2) If $K(t, x)=K \mathbb{1}_{\left[t_{1}, t_{2}\right]}(t)$ with $K \in \mathbb{R}$ and $0 \leq t_{1}<t_{2} \leq T$, then equation (2.4) also has a unique solution $(u, \widetilde{u}) \in C^{0}\left(0, T ; L^{2}(\Omega)\right) \cap L^{2}\left(0, T ; H^{1}(\Omega)\right)$ and

$$
\widetilde{w}(0)=\mathrm{e}^{\left(-K-K^{\prime}\right)\left(t_{2}-t_{1}\right)} w(0) .
$$


(3) If $K(t, x)=K(x)$, with Support $(K) \subset[a, b]$ where $a<b$ and $a \neq 0$ or $b \neq 1$, then equation (2.4-B) is ill-posed: there does not exist in general a solution $\widetilde{u}$, even in the distribution sense.

Theorem 2.4 shows that the BFN algorithm converges and makes the error decrease exponentially if the system is fully observed in space, at least for a subinterval of the time period.

In the following subsection, we prove Theorem 2.4.

\subsection{Proof of Theorem $\mathbf{2 . 4}$}

We refer the reader to [4] for the existence and uniqueness of $u \in C^{0}\left(0, T ; L^{2}(\Omega)\right) \cap L^{2}\left(0, T ; H^{1}(\Omega)\right)$, solution of equation $(2.4-F)$.

\subsubsection{Case 1: $K$ constant}

The difference $w$ satisfies

$$
\left\{\begin{aligned}
\partial_{t} w-\nu \partial_{x x} w+a(x) \partial_{x} w+K w & =0, \\
\left.w\right|_{x=0}=\left.w\right|_{x=1} & =0, \\
\left.w\right|_{t=0} & =w_{0},
\end{aligned}\right.
$$

its existence is ensured by the existence of $u$.

Let us now define $\widetilde{w}$ as in equation (2.7), $w$ is solution of

$$
\left\{\begin{aligned}
\partial_{t} \widetilde{w}-\nu \partial_{x x} \widetilde{w}+a(x) \partial_{x} \widetilde{w}-K^{\prime} \widetilde{w} & =0, \\
\left.\widetilde{w}\right|_{x=0}=\left.\widetilde{w}\right|_{x=1} & =0, \\
\left.\widetilde{w}\right|_{t=T} & =w(T) .
\end{aligned}\right.
$$

And setting $\widetilde{u}=\widetilde{w}+u_{\text {true }}$ proves the existence of $\widetilde{u}$, solution of (2.4-B).

Finally, equation

$$
\left\{\begin{aligned}
\partial_{t} \phi-\nu \partial_{x x} \phi+a(x) \partial_{x} \phi-K^{\prime} \phi & =0 \\
\left.\phi\right|_{x=0}=\left.\phi\right|_{x=1} & =0 \\
\left.\phi\right|_{t=T} & =0,
\end{aligned}\right.
$$

admits $\phi \equiv 0$ as unique solution, and uniqueness for $\widetilde{u}$ follows.

\subsubsection{Case 2: $K(t)$}

We assume that $K(t, x)=K(t)=K \mathbb{1}_{\left[t_{1}, t_{2}\right]}(t)$ with $0 \leq t_{1}<t_{2} \leq T$. Similarly, let us define $\widetilde{w}$ by:

$$
\widetilde{w}(t)= \begin{cases}w(t) \mathrm{e}^{\left(-K-K^{\prime}\right)\left(t_{2}-t_{1}\right)} & \text { if } t \leq t_{1}, \\ w(t) \mathrm{e}^{\left(-K-K^{\prime}\right)\left(t_{2}-t\right)} & \text { if } t_{1}<t<t_{2}, \\ w(t) & \text { if } t \geq t_{2}\end{cases}
$$

We can check that $\widetilde{w}$ is a solution of $(2.9)$, and $\widetilde{u}=\widetilde{w}+u_{\text {true }}$ is a solution of (2.4-B), and as before a uniqueness argument gives the desired result.

\subsubsection{Case 3: $K(x)$}

We now assume that Support $(K) \subset[a, b]$ where $a<b$ and $a \neq 0$ or $b \neq 1$, i.e. the support of $K$ is not $[0,1]$. In that case, there is no solution $\widetilde{w}$ to the backward equation (2.9). In appendix, we give a detailed and technical proof of this in the special case

$$
a(x)=a, \quad K(x)=K \cdot \mathbb{1}_{[0,1 / 2]}(x) .
$$

In the sequel, we give an indication of why this result is true. 
As before, the differences $w$ and $\widetilde{w}$ satisfy equations (2.8) and (2.9). We denote by $S_{+}$and $S_{-}$the dissipative continuous semi-groups of operators in $L^{2}(\Omega)$ associated to equations (2.8) and (2.9), seen as forward equations on $\left[t_{0}, t\right]$ with initial conditions given in $t_{0}$ :

$$
S_{+}\left(t_{0}, t\right)\left(w\left(t_{0}\right)\right)=w(t), \quad S_{-}\left(t_{0}, t\right)\left(\widetilde{w}\left(t_{0}\right)\right)=\widetilde{w}(t) .
$$

The BFN algorithm has a solution if and only if we have

$$
w(T) \in \operatorname{Ran}\left(S_{-}(0, T)\right)
$$

We re-write equation (2.8) associated to $w$ :

$$
\left\{\begin{aligned}
\partial_{t} w-\nu \partial_{x x} w+a(x) \partial_{x} w-K^{\prime} w & =\left(-K-K^{\prime}\right) w \\
\left.w\right|_{x=0}=\left.w\right|_{x=1} & =0 \\
\left.w\right|_{t=0} & =w_{0}
\end{aligned}\right.
$$

so that we have, thanks to Duhamel's formula:

$$
w(t)=S_{-}(0, t)\left(w_{0}\right)+\int_{0}^{t} S_{-}(s, t)\left(\left(-K-K^{\prime}\right) w(s)\right) \mathrm{d} s .
$$

By contradiction, let us assume that the expected result is true for all $T$, i.e. $w(t) \in \operatorname{Ran}\left(S_{-}(0, t)\right)$ for all $t$ :

$$
\forall t, \exists \varphi(t), w(t)=S_{-}(0, t) \varphi(t)
$$

In that case, we replace (2.11) in (2.10) and we get:

$$
w(t)=S_{-}(0, t)\left(w_{0}\right)+\int_{0}^{t} S_{-}(s, t)\left(\left(-K(x)-K^{\prime}(x)\right) S_{-}(0, s) \varphi(s)\right) \mathrm{d} s .
$$

Let us assume, by contradiction, that $-K(x)-K^{\prime}(x)$ commutes with $S_{-}$. Then we get:

$$
S_{-}(0, t)\left(\varphi(t)-w_{0}\right)=\left(-K(x)-K^{\prime}(x)\right) S_{-}(0, t) \int_{0}^{t} \varphi(s) \mathrm{d} s .
$$

But we know that $S_{-}$has the unique continuation property, that is:

Proposition 2.5. If $S_{-}(0, t)(X)=0$ on a non-empty subset of $[0,1]$, then $S_{-}(0, t)(X)=0$ on $[0,1]$.

This result and (2.12) give:

$$
w(t)=S_{-}(0, t)(\varphi(t))=S_{-}(0, t)\left(w_{0}\right)=S_{+}(0, t)\left(w_{0}\right) .
$$

As this stands for every $w_{0}$, we have $S_{-}=S_{+}$and finally $K=K^{\prime}=0$, which is a contradiction. Therefore, $K+K^{\prime}$ does not commute with $S_{-}$. Thus, in general, we cannot find any function $\psi$ such that:

$$
\int_{0}^{t} S_{-}(s, t)\left[\left(-K(x)-K^{\prime}(x)\right) S_{-}(0, s) \varphi(s)\right] \mathrm{d} s=S_{-}(0, t) \psi .
$$




\section{BuRgers' EQUATION With A VISCOUS TERM}

In this section, we consider one step of the BFN algorithm applied to the viscous Burgers' equation:

$$
\begin{aligned}
& (F)\left\{\begin{aligned}
\partial_{t} u-\nu \partial_{x x} u+u \partial_{x} u & =-K\left(u-u_{\mathrm{obs}}\right), \\
\left.u\right|_{x=0}=\left.u\right|_{x=1} & =0, \\
\left.u\right|_{t=0} & =u_{0},
\end{aligned}\right. \\
& (B)\left\{\begin{aligned}
\partial_{t} \widetilde{u}-\nu \partial_{x x} \widetilde{u}+\widetilde{u} \partial_{x} \widetilde{u} & =K^{\prime}\left(\widetilde{u}-u_{\mathrm{obs}}\right), \\
\left.\widetilde{u}\right|_{x=0}=\left.\widetilde{u}\right|_{x=1} & =0, \\
\left.\widetilde{u}\right|_{t=T} & =u(T),
\end{aligned}\right.
\end{aligned}
$$

with the same notations as before.

The observations $u_{\mathrm{obs}}$ are obtained from (2.2)-(2.3), where $u_{\text {true }}$ satisfies the forward Burgers' equation:

$$
\left\{\begin{aligned}
\partial_{t} u_{\text {true }}-\nu \partial_{x x} u_{\text {true }}+u_{\text {true }} \partial_{x} u_{\text {true }} & =0 \\
\left.u_{\text {true }}\right|_{x=0}=\left.u_{\text {true }}\right|_{x=1} & =0 \\
\left.u_{\text {true }}\right|_{t=0} & =u_{\text {true }}^{0}
\end{aligned}\right.
$$

\subsection{Main result and remarks}

Using the previous hypotheses, we recall the following result:

Proposition 3.1. Under Hypotheses 2.2, equation (3.2) has a unique solution $u_{\text {true }} \in C^{0}\left(0, T ; L^{2}(\Omega)\right) \cap$ $L^{2}\left(0, T ; H^{1}(\Omega)\right)$.

We have the following result if $K \neq 0$ :

Theorem 3.2. Under Hypotheses 2.2, let $u_{\text {true }}$ be the solution of (3.2) given by Proposition 3.1, and $u_{\mathrm{obs}}$ given by $(2.2,2.3)$. Then equation (3.1-F) has a unique solution $u \in C^{0}\left(0, T ; L^{2}(\Omega)\right) \cap L^{2}\left(0, T ; H^{1}(\Omega)\right)$. But the Back and Forth problem (3.1-FB) is ill-posed. More specifically, the mapping $u_{0} \mapsto \widetilde{u}(t=0)$ is not $C^{2}$, even when $K(t, x)$ is a constant (except for $K(t, x) \equiv 0)$.

This result, although it is not exactly a non-existence result, is strongly negative, especially in the perspective of numerical applications.

The next subsections are devoted to the proofs of these results.

\subsection{Proof of Theorem 3.2}

For computational simplicity, we assume throughout this section that the observations are identically zero: $u_{\text {obs }}(t, x)=u_{\text {true }}(t, x)=0$ for all $(t, x)$, without loss of generality. Let us first introduce some notations.

Let us denote by $w$ (resp. $\widetilde{w}$ ) the differences between $u$ (resp. $\widetilde{u}$ ) and the true state, as in (2.6), they satisfy the following equations:

$$
\begin{aligned}
(F)\left\{\begin{aligned}
\partial_{t} w-\nu \partial_{x x} w+w \partial_{x} w+K w & =0, \\
\left.w\right|_{x=0}=\left.w\right|_{x=1} & =0, \\
\left.w\right|_{t=0} & =w_{0},
\end{aligned}\right. \\
(B)\left\{\begin{aligned}
\partial_{t} \widetilde{w}-\nu \partial_{x x} \widetilde{w}+\widetilde{w} \partial_{x} \widetilde{w}-K^{\prime} \widetilde{w} & =0, \\
\left.\widetilde{w}\right|_{x=0}=\left.\widetilde{w}\right|_{x=1} & =0, \\
\left.\widetilde{w}\right|_{t=T} & =w(T) .
\end{aligned}\right. \text {. }
\end{aligned}
$$

Let us denote also by $S_{+}$and $S_{-}$the non-linear operators on $L^{2}(\Omega)$ associated to the forward equations with $K$ or $K^{\prime}$ :

$$
S_{+}\left(t_{0}, t\right)\left(w\left(t_{0}\right)\right)=w(t), \quad S_{-}\left(t_{0}, t\right)\left(\widetilde{w}\left(t_{0}\right)\right)=\widetilde{w}(t), \quad \forall t \geq t_{0}
$$


We will also use the dissipative continuous semi-groups of operators in $L^{2}(\Omega), U_{+}$and $U_{-}$, associated to the following linear equations:

$$
\begin{aligned}
& \left\{\begin{array}{rl}
\partial_{t} \phi-\nu \partial_{x x} \phi+K \phi & =0 \\
\left.\phi\right|_{x=0}=\left.\phi\right|_{x=1}=0,\left.\quad \phi\right|_{t=0} & =\phi_{0}
\end{array} \quad \Longleftrightarrow \quad U_{+}(0, t)\left(\phi_{0}\right)=\phi(t),\right.
\end{aligned}
$$

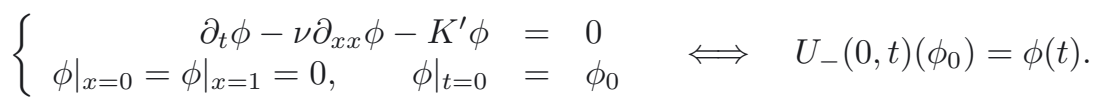

To prove Theorem 3.2 we will prove that $w$ is not in the image of $S_{-}$, in general. To do so we will use perturbations theory. We can easily show that $S_{+}$is infinitely continuous with respect to the data $w_{0}$. So if we suppose that $w_{0}$ is small:

$$
w_{0}=\varepsilon \varphi_{0},
$$

with $\varphi_{0} \in L^{2}(\Omega)$, then we have that $w(t)$, solution of the forward equation (3.3-F) is also small and can be developed in series of $\varepsilon$

$$
w=\varepsilon \sum_{n \geq 0} \varepsilon^{n} w^{n}
$$

Similarly, if we assume that $\widetilde{w}$ is well defined, then we can write as well:

$$
\widetilde{w}=\varepsilon \sum_{n \geq 0} \varepsilon^{n} \widetilde{w}^{n} .
$$

As previously, $w$ satisfies:

$$
\left\{\begin{aligned}
\partial_{t} w-\nu \partial_{x x} w+K w & =-w \partial_{x} w \\
\left.w\right|_{x=0}=\left.w\right|_{x=1} & =0 \\
\left.w\right|_{t=0} & =w_{0},
\end{aligned}\right.
$$

so that if we develop in series of $\varepsilon$ we get, for $w^{0}$ :

$$
\left\{\begin{aligned}
\partial_{t} w^{0}-\nu \partial_{x x} w^{0}+K w^{0} & =0 \\
\left.w^{0}\right|_{x=0}=\left.w^{0}\right|_{x=1} & =0 \\
\left.w^{0}\right|_{t=0} & =\varphi_{0} .
\end{aligned}\right.
$$

For $w^{1}$ we have:

$$
\left\{\begin{aligned}
\partial_{t} w^{1}-\nu \partial_{x x} w^{1}+K w^{1} & =-w^{0} \partial_{x} w^{0} \\
\left.w^{1}\right|_{x=0}=\left.w^{1}\right|_{x=1} & =0 \\
\left.w^{1}\right|_{t=0} & =0 .
\end{aligned}\right.
$$

Similarly we have for $\widetilde{w}^{0}$ and $\widetilde{w}^{1}$ :

$$
\begin{gathered}
\left\{\begin{aligned}
\partial_{t} \widetilde{w}^{0}-\nu \partial_{x x} \widetilde{w}^{0}-K^{\prime} \widetilde{w}^{0} & =0, \\
\left.\widetilde{w}^{0}\right|_{x=0}=\left.\widetilde{w}^{0}\right|_{x=1} & =0, \\
\left.\widetilde{w}^{0}\right|_{t=T} & =w^{0}(T),
\end{aligned}\right. \\
\left\{\begin{aligned}
\partial_{t} \widetilde{w}^{1}-\nu \partial_{x x} \widetilde{w}^{1}-K^{\prime} \widetilde{w}^{1} & =-\widetilde{w}^{0} \partial_{x} \widetilde{w}^{0}, \\
\left.\widetilde{w}^{1}\right|_{x=0}=\left.\widetilde{w}^{1}\right|_{x=1} & =0, \\
\left.\widetilde{w}^{1}\right|_{t=T} & =w^{1}(T) .
\end{aligned}\right.
\end{gathered}
$$

We can compute $w^{0}$ and $w^{1}$ thanks to $U_{+}$:

$$
\begin{aligned}
& w^{0}(t)=U_{+}(0, t)\left(\varphi_{0}\right) \\
& w^{1}(t)=-\int_{0}^{t} U_{+}(s, t)\left[w^{0}(s) \partial_{x} w^{0}(s)\right] \mathrm{d} s .
\end{aligned}
$$


If we assume that $\widetilde{w}^{0}$ is well defined, with

$$
\widetilde{w}^{0}(t)=U_{-}(0, t)\left(\psi_{0}\right)
$$

then the condition $\widetilde{w}(T)=w(T)$ leads to

$$
\begin{aligned}
U_{-}(0, T)\left(\psi_{0}\right) & =U_{+}(0, T)\left(\varphi_{0}\right) \\
\psi_{0} & =\mathrm{e}^{-\left(K+K^{\prime}\right) T} \varphi_{0} .
\end{aligned}
$$

Then we have for $\widetilde{w}^{0}$ :

$$
\begin{aligned}
\widetilde{w}^{0}(t) & =U_{-}(0, t)\left(\psi_{0}\right) \\
& =U_{-}(0, t) \mathrm{e}^{-\left(K+K^{\prime}\right) T} \varphi_{0} .
\end{aligned}
$$

For $\widetilde{w}^{1}$ the final condition $\widetilde{w}^{1}(T)=w^{1}(T)$ gives, thanks to $(3.5)$ :

$$
\begin{aligned}
\widetilde{w}^{1}(T) & =w^{1}(T) \\
& =-\int_{0}^{T} U_{+}(s, t)\left[w^{0}(s) \partial_{x} w^{0}(s)\right] \mathrm{d} s .
\end{aligned}
$$

On the other hand, if we assume that $\widetilde{w}^{1}$ is well defined, with $\widetilde{w}^{1}(0)=\psi_{T}$, then equation (3.4) and the Duhamel formula give

$$
\widetilde{w}^{1}(T)=U_{-}(0, T)\left[\psi_{T}\right]-\int_{0}^{T} U_{-}(s, T)\left[\widetilde{w}^{0}(s) \partial_{x} \widetilde{w}^{0}(s)\right] \mathrm{d} s .
$$

Then, equating (3.9) and (3.8) we should have

$$
U_{-}(0, T)\left[\psi_{T}\right]-\int_{0}^{T} U_{-}(s, T)\left[\widetilde{w}^{0}(s) \partial_{x} \widetilde{w}^{0}(s)\right] \mathrm{d} s=-\int_{0}^{T} U_{+}(s, T)\left[w^{0}(s) \partial_{x} w^{0}(s)\right] \mathrm{d} s .
$$

Therefore

$$
2 U_{-}(0, T)\left[\psi_{T}\right]=\int_{0}^{T} U_{-}(s, T)\left[\partial_{x}\left(\widetilde{w}^{0}(s)^{2}\right)\right] \mathrm{d} s-\int_{0}^{T} U_{+}(s, T)\left[\partial_{x}\left(w^{0}(s)^{2}\right)\right] \mathrm{d} s .
$$

If we assume that $\psi_{T}=\frac{1}{2} \partial_{x} g_{T}$, then we obtain

$$
U_{-}(0, T)\left[g_{T}\right]+c=\int_{0}^{T} U_{-}(s, T)\left[\widetilde{w}^{0}(s)^{2}\right] \mathrm{d} s-\int_{0}^{T} U_{+}(s, T)\left[w^{0}(s)^{2}\right] \mathrm{d} s
$$

where $c$ is a constant. We now use (3.5)-(3.7):

$$
\begin{aligned}
U_{-}(0, T)\left[g_{T}+c \mathrm{e}^{-K^{\prime} T}\right]= & \int_{0}^{T} U_{-}(s, T)\left[U_{-}(0, s)\left(\mathrm{e}^{-\left(K+K^{\prime}\right) T} \varphi_{0}\right)\right]^{2} \mathrm{~d} s \\
& -\int_{0}^{T} U_{+}(s, T)\left[U_{-}(0, s)\left(\varphi_{0}\right)\right]^{2} \mathrm{~d} s \\
= & \left(\mathrm{e}^{-2\left(K+K^{\prime}\right) T}-1\right) \int_{0}^{T} U_{+}(s, T)\left[U_{-}(0, s)\left(\varphi_{0}\right)\right]^{2} \mathrm{~d} s .
\end{aligned}
$$

And if $K>0$ and $K^{\prime}>0$ this last equation can not be solved in general: such $g_{T}$ does not, in general, exist. Indeed, let us do an explicit computation thanks to Fourier series:

$$
\varphi_{0}=\sum_{n \geq 1} a_{n} \mathrm{e}^{\mathrm{i} n x}, \quad g_{T}+c \mathrm{e}^{-K^{\prime} T}=\sum_{n \geq 1} b_{n} \mathrm{e}^{\mathrm{i} n x} .
$$


We recall that we have

$$
\begin{aligned}
& U_{+}(s, t)\left[\sum_{n \geq 1} c_{n} \mathrm{e}^{\mathrm{i} n x}\right]=\sum_{n \geq 1} c_{n} \mathrm{e}^{\mathrm{i} n x} \mathrm{e}^{\left(-K-\nu n^{2}\right)(t-s)}, \\
& U_{-}(s, t)\left[\sum_{n \geq 1} c_{n} \mathrm{e}^{\mathrm{i} n x}\right]=\sum_{n \geq 1} c_{n} \mathrm{e}^{\mathrm{i} n x} \mathrm{e}^{\left(K^{\prime}-\nu n^{2}\right)(t-s)} .
\end{aligned}
$$

Then we can compute the right hand side of equation (3.10):

$$
\begin{aligned}
& \left(\mathrm{e}^{-2\left(K+K^{\prime}\right) T}-1\right) \int_{0}^{T} U_{+}(s, T)\left[\left(U_{-}(0, s)\left(\varphi_{0}\right)\right]^{2} \mathrm{~d} s\right. \\
= & \left(\mathrm{e}^{-2\left(K+K^{\prime}\right) T}-1\right) \int_{0}^{T} U_{+}(s, T)\left[\sum_{n} a_{n} \mathrm{e}^{K^{\prime} s} \mathrm{e}^{\mathrm{i} n x} \mathrm{e}^{-s \nu n^{2}}\right]^{2} \mathrm{~d} s \\
= & \left(\mathrm{e}^{-2\left(K+K^{\prime}\right) T}-1\right) \int_{0}^{T} U_{+}(s, T)\left[\sum_{n} \mathrm{e}^{2 s K^{\prime}} \mathrm{e}^{\mathrm{i} n x} \sum_{p+q=n} a_{p} a_{q} \mathrm{e}^{-s \nu\left(p^{2}+q^{2}\right)}\right] \mathrm{d} s \\
= & \left(\mathrm{e}^{-2\left(K+K^{\prime}\right) T}-1\right) \int_{0}^{T}\left[\sum_{n} \mathrm{e}^{-K(T-s)} \mathrm{e}^{2 s K^{\prime}} \mathrm{e}^{-\nu(T-s) n^{2}} \mathrm{e}^{\mathrm{i} n x} \sum_{p+q=n} a_{p} a_{q} \mathrm{e}^{-s \nu\left(p^{2}+q^{2}\right)}\right] \mathrm{d} s \\
= & \left(\mathrm{e}^{-2\left(K+K^{\prime}\right) T}-1\right) \int_{0}^{T}\left[\sum_{n} \sum_{p+q=n} a_{p} a_{q} \mathrm{e}^{-K T-\nu T n^{2}+\mathrm{i} n x} \mathrm{e}^{2 s K^{\prime}+s K+\nu s n^{2}-s \nu\left(p^{2}+q^{2}\right)}\right] \mathrm{d} s \\
= & \left(\mathrm{e}^{-2\left(K+K^{\prime}\right) T}-1\right)\left[\sum_{n} \sum_{p+q=n} a_{p} a_{q} \mathrm{e}^{-K T-\nu T n^{2}+\mathrm{i} n x} \frac{\mathrm{e}^{2 T K^{\prime}+T K+2 \nu p q T}-1}{2 K^{\prime}+K+2 \nu p q}\right] .
\end{aligned}
$$

For the left hand side of (3.10) we have:

$$
U_{-}(0, T)\left[g_{T}+c \mathrm{e}^{-K^{\prime} T}\right]=\sum_{n} b_{n} \mathrm{e}^{\mathrm{i} n x} \mathrm{e}^{K^{\prime} T-\nu n^{2} T} .
$$

So that we get, for all $n$ :

$$
b_{n}=\mathrm{e}^{\left(-K^{\prime}+K\right) T}\left(\mathrm{e}^{-2\left(K+K^{\prime}\right) T}-1\right)\left[\sum_{p+q=n} a_{p} a_{q} \frac{\mathrm{e}^{2 T K^{\prime}+T K+2 \nu p q T}-1}{2 K^{\prime}+K+2 \nu p q}\right] .
$$

This defines a distribution if and only if $b_{n}$ has polynomial growth, if and only if $\underline{b_{n}}$ has polynomial growth, where

$$
\underline{b_{n}}=\left(\mathrm{e}^{-2\left(K+K^{\prime}\right) T}-1\right)\left[\sum_{p+q=n} a_{p} a_{q} \frac{\mathrm{e}^{2 T K^{\prime}+T K+2 \nu p q T}}{2 K^{\prime}+K+2 \nu p q}\right],
$$

which is clearly not the case for every sequence $\left(a_{n}\right)$ with polynomial growth, unless $K=K^{\prime}=0$.

Therefore, the mapping $w_{(t=0)} \mapsto \widetilde{w}(t=0)$ is not $C^{2}$.

\section{Non Viscous tRANSPORT EQUATIONS}

We consider here the extension of Theorem 2.4 to the inviscid case, for both linear transport and Burgers' equations. 


\subsection{Inviscid linear transport}

We first consider the linear case. The BFN equations are:

$$
\begin{aligned}
& (F)\left\{\begin{aligned}
\partial_{t} u+a(x) \partial_{x} u & =-K\left(u-u_{\mathrm{obs}}\right), \\
\left.u\right|_{x=0} & =\left.u\right|_{x=1}, \\
\left.u\right|_{t=0} & =u_{0}
\end{aligned}\right. \\
& (B)\left\{\begin{aligned}
\partial_{t} \widetilde{u}+a(x) \partial_{x} \widetilde{u} & =K^{\prime}\left(\widetilde{u}-u_{\mathrm{obs}}\right), \\
\left.\widetilde{u}\right|_{x=0} & =\left.\widetilde{u}\right|_{x=1}, \\
\left.\widetilde{u}\right|_{t=T} & =u(T),
\end{aligned}\right.
\end{aligned}
$$

and $u_{\text {obs }}$ given by $(2.2,2.3)$, with

$$
\left\{\begin{aligned}
\partial_{t} u_{\text {true }}+a(x) \partial_{x} u_{\text {true }} & =0 \\
\left.u_{\text {true }}\right|_{x=0} & =\left.u_{\text {true }}\right|_{x=1} \\
\left.u_{\text {true }}\right|_{t=0} & =u_{\text {true }}^{0}
\end{aligned}\right.
$$

with the following hypotheses:

\section{Hypotheses 4.1.}

- $K$ and $K^{\prime} \in C^{1}([0 ; T] \times \Omega)$ are positive and may depend on $t$ and $x$, but for the sake of simplicity, we will always assume that there exists a constant $\kappa \in \mathbb{R}_{+}^{*}$ such that $K^{\prime}(t, x)=\kappa K(t, x)$;

- $a(x) \in C^{1}(\Omega)$;

- $u_{\mathrm{true}}^{0} \in C^{1}(\Omega)$ and $u_{0} \in C^{1}(\Omega)$, both periodic in space.

Proposition 4.2. Under Hypotheses 4.1, equation (4.2) has a unique classical solution $u_{\text {true }} \in C^{1}([0 ; T] \times \Omega)$.

We denote by

$$
(s, \psi(s, x))
$$

the characteristic curve of equation $(4.1-F)$ with $K=0$, with foot $x$ at time $s=0$, i.e. such that

$$
\left.(s, \psi(s, x))\right|_{s=0}=(0, x) .
$$

We recall that the characteristics are well defined and do not intersect over $[0, T]$.

Proof. Let $(s, x) \in[0, T] \times \Omega$. Following the method of characteristics, we notice that, if $\psi(s, x)=y$, then

$$
y=\psi(s, x)=x+s a(y) .
$$

We now compute

$$
\frac{\mathrm{d}}{\mathrm{d} t} u(t, x+t a(y))=\partial_{t} u(t, x+t a(y))+a(y) \partial_{x} u(t, x+t a(y))=-K\left(u-u_{\mathrm{obs}}\right)(t, x+t a(y)) .
$$

Thus

Therefore

$$
\frac{\mathrm{d}}{\mathrm{d} t} u(t, x+t a(y))+K(t, x+t a(y)) u(t, x+t a(y))=K(t, x+t a(y)) u_{\mathrm{obs}}(t, x+t a(y)) .
$$

$$
\begin{aligned}
u(t, x+t a(y))= & u(0, x) \exp \left(-\int_{0}^{t} K(s, x+s a(y)) \mathrm{d} s\right) \\
& +\int_{0}^{t} \exp \left(-\int_{s}^{t} K(\sigma, x+\sigma a(y)) \mathrm{d} \sigma\right) K(s, x+s a(y)) u_{\mathrm{obs}}(s, x+s a(y)) \mathrm{d} s .
\end{aligned}
$$


Theorem 4.3. Under Hypotheses 4.1, let $u_{\text {true }}$ be the classical solution of (4.2) given by Proposition 4.2, and $u_{\mathrm{obs}}$ given by (2.2)-(2.3). Then equations $(4.1-F)$ and $(4.1-B)$ have unique classical solutions $u$ and $\widetilde{u} \in C^{1}([0 ; T] \times \Omega)$. We denote

Then we have:

$$
\begin{aligned}
& w(t)=u(t)-u_{\text {true }}(t), \\
& \widetilde{w}(t)=\widetilde{u}(t)-u_{\text {true }}(t) .
\end{aligned}
$$

(1) If $K(t, x)=K$, then for all $t \in[0, T]$ :

$$
\widetilde{w}(t)=w(t) \mathrm{e}^{\left(-K-K^{\prime}\right)(T-t)} .
$$

(2) If $K(t, x)=K \mathbb{1}_{\left[t_{1}, t_{2}\right]}(t)$ with $0 \leq t_{1}<t_{2} \leq T$, then

$$
\widetilde{w}(0)=w(0) \mathrm{e}^{\left(-K-K^{\prime}\right)\left(t_{2}-t_{1}\right)} .
$$

(3) If $K(t, x)=K(x)$, then for all $t \in[0, T]$ :

$$
\widetilde{w}(t, \psi(t, x))=w(t, \psi(t, x)) \exp \left(-\int_{t}^{T} K(\psi(s, x))+K^{\prime}(\psi(s, x)) \mathrm{d} s\right) .
$$

\subsection{Non viscous Burgers' equation}

We finally consider non viscous Burgers' equation, once again with periodic boundary conditions, and for a time $T$ such that there is no shock in the interval $[0, T]$ :

$$
\begin{aligned}
& (F)\left\{\begin{aligned}
\partial_{t} u+u \partial_{x} u & =-K\left(u-u_{\mathrm{obs}}\right), \\
\left.u\right|_{x=0} & =\left.u\right|_{x=1}, \\
\left.u\right|_{t=0} & =u_{0}
\end{aligned}\right. \\
& (B)\left\{\begin{aligned}
\partial_{t} \widetilde{u}+\widetilde{u} \partial_{x} \widetilde{u} & =K^{\prime}\left(\widetilde{u}-u_{\mathrm{obs}}\right), \\
\left.\widetilde{u}\right|_{x=0} & =\left.\widetilde{u}\right|_{x=1}, \\
\left.\widetilde{u}\right|_{t=T} & =u(T),
\end{aligned}\right.
\end{aligned}
$$

where $u_{\mathrm{obs}}$ is given by $(2.2)-(2.3)$, with

$$
\left\{\begin{aligned}
\partial_{t} u_{\text {true }}+u_{\text {true }} \partial_{x} u_{\text {true }} & =0 \\
\left.u_{\text {true }}\right|_{x=0} & =\left.u_{\text {true }}\right|_{x=1} \\
\left.u_{\text {true }}\right|_{t=0} & =u_{\text {true }}^{0}
\end{aligned}\right.
$$

with the following hypotheses:

\section{Hypotheses 4.4.}

- $K$ and $K^{\prime} \in C^{1}([0 ; T] \times \Omega)$ are positive and may depend on $t$ and $x$, but for the sake of simplicity, we will always assume that there exists a constant $\kappa \in \mathbb{R}_{+}^{*}$ such that $K^{\prime}(t, x)=\kappa K(t, x)$;

- $u_{\text {true }}^{0} \in C^{1}(\Omega)$ and $u_{0} \in C^{1}(\Omega)$, both periodic in space;

- If $\exists x \in \Omega$ such that $u_{0}^{\prime}(x)<0$, then we assume that $T<T^{*}=\left[\max _{x \in \Omega}\left(-u_{0}^{\prime}(x)\right)\right]^{-1}$, otherwise $T \in] 0,+\infty[$.

Proposition 4.5. Under Hypotheses 4.4, equation (4.7) has a unique classical solution $u_{\text {true }} \in C^{1}([0 ; T] \times \Omega)$.

Theorem 4.6. Under Hypotheses 4.4, let $u_{\text {true }}$ be the classical solution of (4.7) given by Proposition 4.5, and $u_{\mathrm{obs}}$ given by $(2.2,2.3)$. Then equations $(4.6-F)$ and $(4.6-B)$ have unique classical solutions $u$ and $\widetilde{u} \in C^{1}([0 ; T] \times \Omega)$. We denote

$$
\begin{aligned}
& w(t)=u(t)-u_{\text {true }}(t), \\
& \widetilde{w}(t)=\widetilde{u}(t)-u_{\text {true }}(t) .
\end{aligned}
$$


Let

Then we have:

$$
M=\sup _{(t, x) \in[0, T] \times \Omega}\left|\partial_{x} u_{\text {true }}(t, x)\right|<+\infty .
$$

(1) If $K(t, x)=K$, then for all $t \in[0, T]$ :

$$
\|\widetilde{w}(t)\|_{L^{2}(\Omega)} \leq \mathrm{e}^{\left(-K-K^{\prime}+M\right)(T-t)}\|w(t)\|_{L^{2}(\Omega)} .
$$

(2) If $K(t, x)=K \mathbb{1}_{\left[t_{1}, t_{2}\right]}(t)$ with $0 \leq t_{1}<t_{2} \leq T$, then

$$
\|\widetilde{w}(0)\|_{L^{2}(\Omega)} \leq \mathrm{e}^{\left(-K-K^{\prime}\right)\left(t_{2}-t_{1}\right)+M T}\|w(0)\|_{L^{2}(\Omega)} .
$$

We also have the following result:

Proposition 4.7. We consider one forward (resp. backward) BFN step of the non viscous Burgers equation (4.6-F) (resp. (4.6-B)). With the notations of Theorem 4.6, if $K(t, x)=K(x)$, then we have

$$
w(T, \psi(T, x))=w(0, x) \exp \left(-\int_{0}^{T} K(\psi(\sigma, x)) \mathrm{d} \sigma-\int_{0}^{T} \partial_{x} u_{\text {true }}(\sigma, \psi(\sigma, x)) \mathrm{d} \sigma\right) .
$$

\subsection{Remarks}

Remark 4.8. For the special case $K(t, x)=K(x)=K \mathbb{1}_{[a, b]}(x)$ where $K$ is a constant and $[a, b]$ is a non-empty sub-interval of $[0,1]$, we have

$$
w(T, \psi(T, x))=w(0, x) \exp \left(-K \chi(x)-\int_{0}^{T} \partial_{x} u_{\text {true }}(\sigma, \psi(\sigma, x)) \mathrm{d} \sigma\right),
$$

where

$$
\chi(x)=\int_{0}^{T} \mathbb{1}_{[a, b]}(\psi(\sigma, x)) \mathrm{d} \sigma
$$

is the time during which the characteristic curve $\psi(\sigma, x)$ with foot $x$ of equation $(4.6-F)$ with $K=0$ lies in the the support of $K$. The system is then observable if and only if the function $\chi$ has a non-zero lower bound, i.e. $m:=\min _{x} \chi(x)>0$, the observability being defined by (see [18]):

$$
\exists C, \forall u \text { solution of }(4.6-F) \text { with } K=0, \quad\|u(T, .)\|^{2} \leq C \int_{0}^{T}\|K(.) u(s, .)\|^{2} \mathrm{~d} s .
$$

In this case, Proposition 4.7 proves the global exponential decrease of the error, provided $K$ is larger than $\frac{M T}{m}$, where $M$ is defined by equation (4.8).

From Remark 4.8, we can easily deduce that if for each iteration, both in the forward and backward integrations, the observability condition is satisfied, then the algorithm converges. Note that this is not a necessary condition, as even if $\chi(x)=0$, the last exponential of equation (4.12) is bounded.

Note also that in real geophysical applications (either meteorology or oceanography), there is usually no viscosity. In this case, assuming the observability condition, the BFN algorithm is well posed, and Theorem 4.6 and Proposition 4.7 say that the solution tends to the observation trajectory everywhere, and not only on the support of $K$. From a numerical point of view, we can observe that even with discrete and sparse observations in space, the numerical solution is corrected everywhere [2]. We also observed that with a not too large viscosity coefficient, the behavior of the algorithm remains unchanged. 

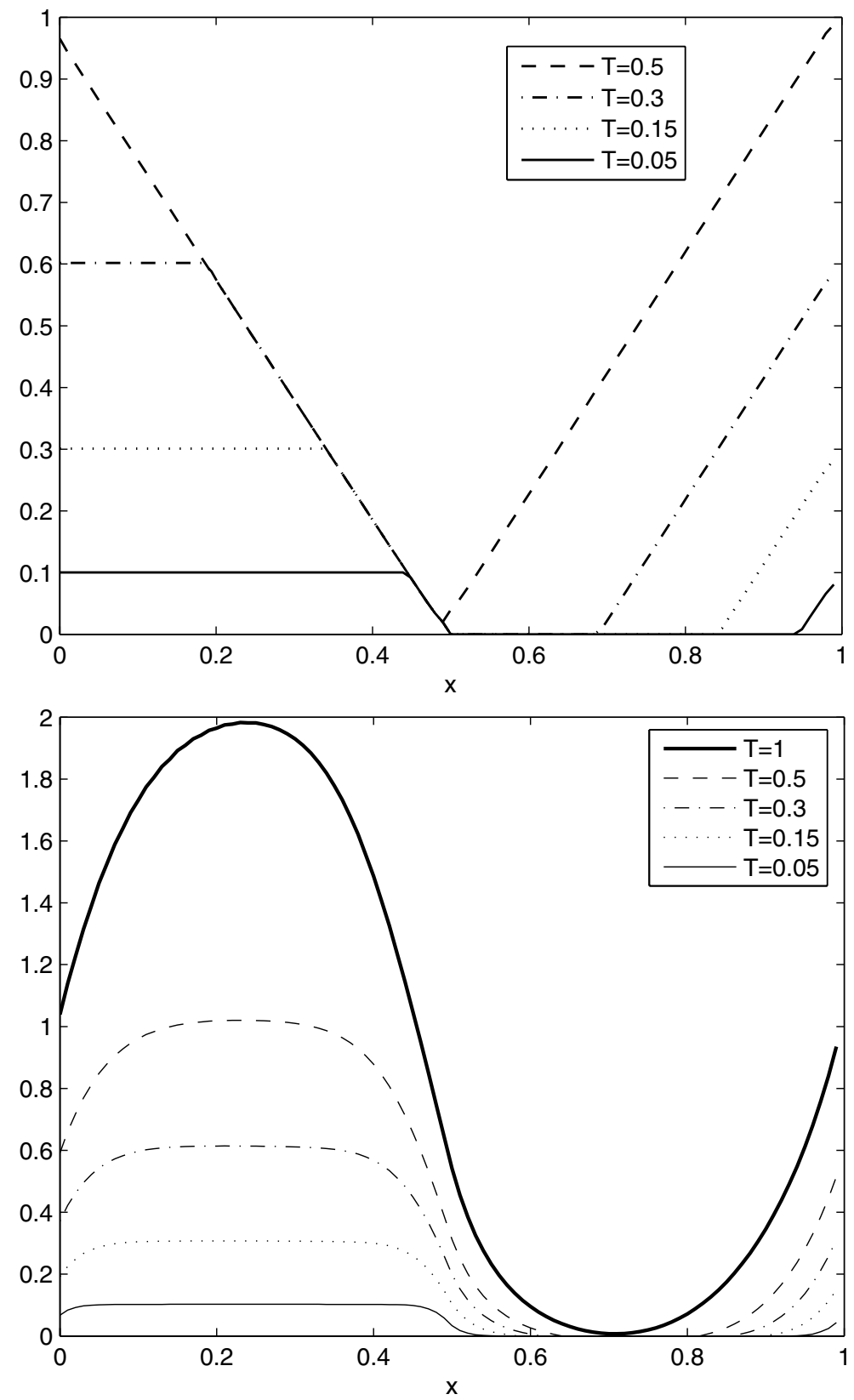

Figure 1. Rate of decay of the error after one iteration of BFN (see equation (4.14)) as a function of $x$, for various times $T$; top: linear transport equation; bottom: inviscid Burgers' equation.

Figure 1 illustrates the results given in Theorem 4.3 in the case 3 (top) and Proposition 4.7 and Remark 4.8 (bottom). These numerical results correspond to a simple case: $u_{\text {true }} \equiv 0, u_{0}(x)=\alpha \sin (2 \pi x), K=K^{\prime}=$ 
$\mathbb{1}_{[0 ; 0.5]}(x)$. Various final times $T$ are considered, from 0.05 to 1 , and both figures show the following expression

$$
-\log \left(\frac{\widetilde{w}(0, x)}{w(0, x)}\right)
$$

as a function of $x \in[0 ; 1]$. Figure 1-top illustrates equation (4.5). The best possible rate of decay is then $\max \left(K+K^{\prime}\right) \times T=2 T$. In the linear case, the transport is $a(x) \equiv 1$. As half of the domain is observed, the observability condition is satisfied if and only if $T>0.5$, and this is confirmed by the figure. Concerning Burgers' equation, Figure 1-bottom illustrates equation (4.11). After one iteration of BFN, the best possible rate of decay is also $2 T$. We can see that in this case, due to the nonlinearities of the model, the solution is less corrected on $[0 ; 0.1]$ but more on $[0.5 ; 0.6]$. From this figure, we can see that the observability condition is satisfied for $T$ larger than approximately 1.

The next subsections are devoted to the proofs of these results.

\subsection{Linear case: Proof of Theorem 4.3}

The first two points of the theorem are easily proven as in Theorem 2.4 with a vanishing viscosity.

Thus we only prove the third point. To do so, we recall that the curves $(s, \psi(s, x))$ are the characteristics of the direct equation $(4.1-F)$ with $K=0$, such that $\left.(s, \psi(s, x))\right|_{s=0}=(0, x)$ (see $[5,6]$ for characteristics theory).

For the forward equation (4.1- $F)$, this change of variable gives

$$
\partial_{s} w(s, \psi(s, x))=-K(\psi(s, x)) w(s, \psi(s, x)) .
$$

So that

$$
w(s, \psi(s, x))=w(0, x) \exp \left(-\int_{0}^{s} K(\psi(\sigma, x)) \mathrm{d} \sigma\right) .
$$

And in particular for $w(T)$ we have

$$
w(T, \psi(T, x))=w(0, x) \exp \left(-\int_{0}^{T} K(\psi(\sigma, x)) \mathrm{d} \sigma\right) .
$$

For $\widetilde{w}$ we have similarly

$$
\partial_{s} \widetilde{w}(s, \psi(s, x))=K^{\prime}(\psi(s, x)) \widetilde{w}(s, \psi(s, x)) .
$$

So that we have:

$$
\begin{aligned}
\widetilde{w}(s, \psi(s, x)) & =\widetilde{w}(T, \psi(T, x)) \exp \left(-\int_{s}^{T} K^{\prime}(\psi(\sigma, x)) \mathrm{d} \sigma\right) \\
& =w(T, \psi(T, x)) \exp \left(-\int_{s}^{T} K^{\prime}(\psi(\sigma, x)) \mathrm{d} \sigma\right) .
\end{aligned}
$$

Using (4.16) and (4.15) we get

$$
\begin{aligned}
& \widetilde{w}(s, \psi(s, x)) \\
= & w(0, x) \exp \left(-\int_{0}^{T} K(\psi(\sigma, x)) \mathrm{d} \sigma\right) \exp \left(-\int_{s}^{T} K^{\prime}(\psi(\sigma, x)) \mathrm{d} \sigma\right) \\
= & w(s, \psi(s, x)) \exp \left(\int_{0}^{s} K(\psi(\sigma, x)) \mathrm{d} \sigma\right) \exp \left(-\int_{0}^{T} K(\psi(\sigma, x)) \mathrm{d} \sigma\right) \exp \left(-\int_{s}^{T} K^{\prime}(\psi(\sigma, x)) \mathrm{d} \sigma\right) \\
= & w(s, \psi(s, x)) \exp \left(-\int_{s}^{T} K(\psi(\sigma, x))+K^{\prime}(\psi(\sigma, x)) \mathrm{d} \sigma\right) .
\end{aligned}
$$




\subsection{Non linear case: Proofs of Theorem 4.6 and Proposition 4.7}

From equation (4.6), we deduce that the forward error $w$ satisfies the following equation:

$$
\partial_{t} w+w \partial_{x} w+u_{\text {true }} \partial_{x} w+w \partial_{x} u_{\text {true }}=-K w .
$$

By multiplying by $w$ and integrating over $\Omega$, we obtain

$$
\frac{1}{2} \partial_{t}\left(\int_{\Omega} w^{2}\right)+\int_{\Omega} w^{2} \partial_{x} w+\int_{\Omega}\left(u_{\text {true }} w \partial_{x} w+w^{2} \partial_{x} u_{\text {true }}\right)=-\int_{\Omega} K w^{2} .
$$

Some integrations by part give the following:

$$
\partial_{t}\left(\|w(t)\|^{2}\right)=\int_{\Omega}\left(-2 K-\partial_{x} u_{\text {true }}\right) w^{2} .
$$

We set $M=\left\|\partial_{x} u_{\text {true }}\right\|_{\infty}$, and as $K$ does not depend on $x$,

$$
\partial_{t}\left(\|w(t)\|^{2}\right) \leq(-2 K+M)\|w(t)\|^{2} .
$$

We have a similar result for the backward error:

$$
\partial_{t}\left(\|\widetilde{w}(t)\|^{2}\right) \leq\left(-2 K^{\prime}+M\right)\|\widetilde{w}(t)\|^{2} .
$$

We first consider the first point of Theorem 4.6, i.e. $K(t, x)=K$. Grönwall's lemma between times $t$ and $T$ gives

$$
\begin{aligned}
\|w(T)\|^{2} & \leq \mathrm{e}^{(-2 K+M)(T-t)}\|w(t)\|^{2}, \\
\|\widetilde{w}(t)\|^{2} & \leq \mathrm{e}^{\left(-2 K^{\prime}+M\right)(T-t)}\|\widetilde{w}(T)\|^{2},
\end{aligned}
$$

from which equation (4.9) is easily deduced.

In the second case, i.e. $K(t, x)=K \mathbb{1}_{\left[t_{1}, t_{2}\right]}(t)$ and by successively applying Grönwall's lemma between times 0 and $t_{1}, t_{1}$ and $t_{2}$, and $t_{2}$ and $T$, one obtains equation (4.10).

Finally, in the case $K(t, x)=K(x)$, by considering a similar approach as in Section 4.1, i.e. using the characteristics of the direct equation $(4.6-F)$ (resp. B), it is straightforward to prove that

$$
w(s, \psi(s, x))=w(0, x) \mathrm{e}^{-\int_{0}^{s} K(\psi(\sigma, x)) \mathrm{d} \sigma-\int_{0}^{s} \partial_{x} u_{\text {true }}(\sigma, \psi(\sigma, x)) \mathrm{d} \sigma},
$$

and then,

$$
w(T, \psi(T, x))=w(0, x) \mathrm{e}^{-\int_{0}^{T} K(\psi(\sigma, x)) \mathrm{d} \sigma} \mathrm{e}^{-\int_{0}^{T} \partial_{x} u_{\text {true }}(\sigma, \psi(\sigma, x)) \mathrm{d} \sigma},
$$

from which equation (4.11) is easily deduced.

\section{Conclusion}

Several conclusions can be drawn from all these results. First of all, in many situations, the coupled forwardbackward problem is well posed, and the nudging terms allow the solution to be corrected (towards the observation trajectory) everywhere and with an exponential convergence. From a numerical point of view, these results have been observed in several geophysical situations, and many numerical experiments have confirmed the global convergence of the BFN algorithm [2]. 
The second remark is that the worst situation, i.e. for which there is no solution to the BFN problem, is the viscous Burgers' equation. But in real geophysical applications, there is most of the time no theoretical viscosity in the equation, and one should consider the inviscid equation instead, for which some convergence results are given. From the numerical point of view, these phenomena are easily confirmed, as well as the exponential decrease of the error $w$. But we also noticed that if the observations are not too sparse, the algorithm works well even with a quite large viscosity.

Finally, these results extend the theory of linear observers in automatics [15]: instead of considering an infinite time interval (only one forward equation but for $T \rightarrow+\infty$ ), one can consider an infinite number of BFN iterations on a finite time interval. This is of great interest in almost all real applications, for which it is not possible to consider a very large time period.

\section{Appendix: Spectral COMputations}

Here we give details for the proof of Theorem 2.4 (case $3: K(x))$ for the example case

$$
a(x)=a, \quad K(x)=K \cdot \mathbb{1}_{[0,1 / 2]}(x) .
$$

We will study the following equation:

$$
\left\{\begin{aligned}
\partial_{t} w-\nu \partial_{x x} w+a \partial_{x} w+K \cdot \mathbb{1}_{[0,1 / 2]}(x) w & =0 \\
\left.w\right|_{x=0}=\left.w\right|_{x=1} & =0 \\
\left.w\right|_{t=0} & =w_{0}
\end{aligned}\right.
$$

To do so, we are going to compute the spectrum of the following operator:

$$
\left\{\begin{array}{l}
A_{K}(u)=-\nu \partial_{x x} u+a \partial_{x} u+K \cdot \mathbb{1}_{[0,1 / 2]}(x) u, \\
\left.u\right|_{x=0}=\left.u\right|_{x=1}=0, \\
\lim _{x \rightarrow \frac{1}{2}, x<\frac{1}{2}} u(x)=\lim _{x \rightarrow \frac{1}{2}, x>\frac{1}{2}} u(x), \\
\lim _{x \rightarrow \frac{1}{2}, x<\frac{1}{2}} \partial_{x} u(x)=\lim _{x \rightarrow \frac{1}{2}, x>\frac{1}{2}} \partial_{x} u(x) .
\end{array}\right.
$$

\subsection{First examples}

We will first consider two simple cases, to explain how we will proceed. First, let us consider the well-known case $a=K=0$, corresponding to the Laplacian operator with Dirichlet conditions on $[0,1]$ :

$$
\left\{\begin{array}{l}
A(u)=-\nu \partial_{x x} u \\
\left.u\right|_{x=0}=\left.u\right|_{x=1}=0 .
\end{array}\right.
$$

If we look for eigenfunctions of the type $u(x)=\mathrm{e}^{\mathrm{i} k x}$ with $\lambda$ as associated eigenvalue, we obtain $u(x)=$ $c_{+} \mathrm{e}^{\mathrm{i} k x}+c_{-} \mathrm{e}^{-\mathrm{i} k x}$, with $k=\sqrt{\frac{\lambda}{\nu}}$ and $k=-\sqrt{\frac{\lambda}{\nu}}$. Then the Dirichlet conditions give

$$
\left\{\begin{array}{l}
c_{+}+c_{-}=0 \\
c_{+} \mathrm{e}^{\mathrm{i} k}+c_{-} \mathrm{e}^{-\mathrm{i} k}=0
\end{array}\right.
$$

and this has a non trivial solution if and only if the following determinant is zero:

$$
\left|\begin{array}{ll}
1 & 1 \\
\mathrm{e}^{\mathrm{i} k} & \mathrm{e}^{-\mathrm{i} k}
\end{array}\right|=0,
$$


which gives

$$
\mathrm{e}^{-\mathrm{i} k}-\mathrm{e}^{\mathrm{i} k}=0=-2 \mathrm{i} \sin (k),
$$

therefore

$$
k=m \pi, m \in \mathbb{Z}, \quad \lambda=\nu \pi^{2} m^{2}, m \in \mathbb{Z} .
$$

Now let us do exactly the same with $K=0$ and $a \neq 0$. The operator reads

$$
\left\{\begin{array}{l}
A(u)=-\nu \partial_{x x} u+a \partial_{x} u \\
\left.u\right|_{x=0}=\left.u\right|_{x=1}=0
\end{array}\right.
$$

Similarly, eigenfunctions of the type $u(x)=\mathrm{e}^{\mathrm{i} k x}$ lead to $u=c_{+} \mathrm{e}^{\mathrm{i} k_{+} x}+c_{-} \mathrm{e}^{i k_{-} x}$, with $k_{ \pm}=\frac{-\mathrm{i} a \pm \sqrt{4 \nu \lambda-a^{2}}}{2 \nu}$. Dirichlet conditions lead to:

$$
\left\{\begin{array}{l}
c_{+}+c_{-}=0 \\
c_{+} \mathrm{e}^{\mathrm{i} k_{+}}+c_{-} \mathrm{e}^{\mathrm{i} k_{-}}=0
\end{array}\right.
$$

so that

$$
0=\mathrm{e}^{\mathrm{i} k_{+}}-\mathrm{e}^{\mathrm{i} k_{-}}=\mathrm{e}^{\mathrm{i} \frac{k_{+}+k_{-}}{2}}\left(\mathrm{e}^{\mathrm{i} \frac{k_{+}-k_{-}}{2}}-\mathrm{e}^{-\mathrm{i} \frac{k_{+}-k_{-}}{2}}\right)=2 \mathrm{i}^{\frac{a}{2 \nu}} \sin \left(\frac{\sqrt{4 \nu \lambda-a^{2}}}{2 \nu}\right) .
$$

Therefore

which leads to

$$
\frac{\sqrt{4 \nu \lambda-a^{2}}}{2 \nu}=\pi m, m \in \mathbb{Z}
$$

$$
\lambda=\nu \pi^{2} m^{2}+\frac{a^{2}}{4 \nu}, m \in \mathbb{Z}, \quad k_{ \pm}=-\frac{\mathrm{i} a}{2 \nu} \pm \pi m, m \in \mathbb{Z} .
$$

\subsection{General case}

Let us now move to the general case $a \neq 0, K \neq 0$. We proceed similarly. Let us recall that

$$
A_{K}=-\nu \partial_{x x}+a \partial_{x}+K \cdot \mathbb{1}_{[0,1 / 2]}(x) .
$$

Looking for eigenfunctions of the type $u(x)=\mathrm{e}^{\mathrm{i} k x}$ leads to different equations for $k$, depending on $x \in[0,1 / 2]$ or $x \in[1 / 2,1]$ :

with roots

$$
\nu k_{1}^{2}+\mathrm{i} a k_{1}+K=\lambda, \quad \nu k_{2}^{2}+\mathrm{i} a k_{2}=\lambda,
$$

$$
k_{1}^{ \pm}=\frac{-\mathrm{i} a \pm \sqrt{4 \nu \lambda-a^{2}-4 \nu K}}{2 \nu}, \quad k_{2}^{ \pm}=\frac{-\mathrm{i} a \pm \sqrt{4 \nu \lambda-a^{2}}}{2 \nu} .
$$

Remark 6.1. As we are interested in the asymptotics $\lambda \rightarrow \infty$, we do not have to consider the case $\Delta_{i}<0$.

We then look for eigenfunctions of the type:

$$
u(x)=\left(c_{1}^{+} \mathrm{e}^{\mathrm{i} k_{1}^{+} x}+c_{1}^{-} \mathrm{e}^{\mathrm{i} k_{1}^{-} x}\right) \cdot \mathbb{1}_{\left[0, \frac{1}{2}\right]}(x)+\left(c_{2}^{+} \mathrm{e}^{\mathrm{i} k_{2}^{+} x}+c_{2}^{-} \mathrm{e}^{\mathrm{i} k_{2}^{-} x}\right) \cdot \mathbb{1}_{\left[\frac{1}{2}, 1\right]}(x) .
$$

Then the conditions read:

$$
\left\{\begin{array}{l}
0=u(0)=c_{1}^{+}+c_{1}^{-} \\
0=u(1)=c_{2}^{+} \mathrm{e}^{\mathrm{i} k_{2}^{+}}+c_{2}^{-} \mathrm{e}^{\mathrm{i} k_{2}^{-}}, \\
0=u\left(\left(\frac{1}{2}\right)^{-}\right)-u\left(\left(\frac{1}{2}\right)^{+}\right)=c_{1}^{+} \mathrm{e}^{\mathrm{i} k_{1}^{+} / 2}+c_{1}^{-} \mathrm{e}^{\mathrm{i} k_{1}^{-} / 2}-c_{2}^{+} \mathrm{e}^{\mathrm{i} k_{2}^{+} / 2}-c_{2}^{-} \mathrm{e}^{\mathrm{i} k_{2}^{-} / 2}, \\
0=\partial_{x} u\left(\left(\frac{1}{2}\right)^{-}\right)-\partial_{x} u\left(\left(\frac{1}{2}\right)^{+}\right)=\mathrm{i} k_{1}^{+} c_{1}^{+} \mathrm{e}^{\mathrm{i} k_{1}^{+} / 2}+\mathrm{i} k_{1}^{-} c_{1}^{-} \mathrm{e}^{\mathrm{i} k_{1}^{-} / 2}-\mathrm{i} k_{2}^{+} c_{2}^{+} \mathrm{e}^{\mathrm{i} k_{2}^{+} / 2}-\mathrm{i} k_{2}^{-} c_{2}^{-} \mathrm{e}^{\mathrm{i} k_{2}^{-} / 2},
\end{array}\right.
$$


which has a non trivial solution if and only if

$$
\sin \left(\frac{k_{1}^{-}-k_{1}^{+}}{4}\right) \cdot\left(k_{2}^{-} \mathrm{e}^{\mathrm{i} k_{2}^{+} / 2}-k_{2}^{+} \mathrm{e}^{\mathrm{i} k_{2}^{-} / 2}\right)+\sin \left(\frac{k_{2}^{-}-k_{2}^{+}}{4}\right) \cdot\left(k_{1}^{-} \mathrm{e}^{\mathrm{i} k_{1}^{-} / 2}-k_{1}^{+} \mathrm{e}^{\mathrm{i} k_{1}^{+} / 2}\right)=0 .
$$

We rewrite the previous equation using

$$
k_{1}^{ \pm}=\frac{-\mathrm{i} a}{2 \nu} \pm l_{1}, \quad k_{2}^{ \pm}=\frac{-\mathrm{i} a}{2 \nu} \pm l_{2},
$$

with

$$
l_{1}=\frac{\sqrt{4 \nu \lambda-a^{2}-4 \nu K}}{2 \nu}, \quad l_{2}=\frac{\sqrt{4 \nu \lambda-a^{2}}}{2 \nu} .
$$

We have $k_{2}^{-} \mathrm{e}^{\mathrm{i} k_{2}^{+} / 2}-k_{2}^{+} \mathrm{e}^{\mathrm{i} k_{2}^{-} / 2}=2 \mathrm{e}^{\frac{a}{4 \nu}}\left(\frac{a}{2 \nu} \sin \left(\frac{l_{2}}{2}\right)-l_{2} \cos \left(\frac{l_{2}}{2}\right)\right)$.

Similarly, $k_{1}^{-} \mathrm{e}^{\mathrm{i} k_{1}^{-} / 2}-k_{1}^{+} \mathrm{e}^{\mathrm{i} k_{1}^{+} / 2}=-2 \mathrm{e}^{\frac{a}{4 \nu}}\left(\frac{a}{2 \nu} \sin \left(\frac{l_{1}}{2}\right)+l_{1} \cos \left(\frac{l_{1}}{2}\right)\right)$. Then (6.4) reads as

$$
l_{2} \sin \left(\frac{l_{1}}{2}\right) \cos \left(\frac{l_{2}}{2}\right)+l_{1} \sin \left(\frac{l_{2}}{2}\right) \cos \left(\frac{l_{1}}{2}\right)=0 .
$$

For large values of $\lambda$, (6.6) gives $l_{1} \sim l_{2} \sim \sqrt{\frac{\lambda}{\nu}}$, and thanks to (6.7) we get $l_{1} \sim l_{2} \sim \pi m$, with $m \in \mathbb{Z}^{*}$. We will denote $l_{1, m}$ and $l_{2, m}$ the solutions of equation (6.7) equivalent to $\pi m$, for $m \in \mathbb{Z}^{*}, k_{1, m}^{ \pm}, k_{2, m}^{ \pm}$the associated roots, $\lambda_{m}$ the eigenvalues, and $e_{m}$ the eigenvectors.

Let us now compute the eigenvectors of $A_{K}$. As we saw, they can be written as

$$
e_{m}=\left(c_{1}^{+} \mathrm{e}^{\mathrm{i} k_{1}^{+} x}+c_{1}^{-} \mathrm{e}^{\mathrm{i} k_{1}^{-} x}\right) \cdot \mathbb{1}_{\left[0, \frac{1}{2}\right]}(x)+\left(c_{2}^{+} \mathrm{e}^{\mathrm{i} k_{2}^{+} x}+c_{2}^{-} \mathrm{e}^{\mathrm{i} k_{2}^{-} x}\right) \cdot \mathbb{1}_{\left[\frac{1}{2}, 1\right]}(x)
$$

(at the moment, we omit $m$ for readability).

The first condition in (6.3) is $c_{1}^{+}+c_{1}^{-}=0$, so that we get $c_{1}^{-}=-c_{1}^{+}$. Setting $c_{1}=2 \mathrm{i} c_{1}^{+}$and using (6.5), we can rewrite $e_{m}$ as:

$$
e_{m}=c_{1} \mathrm{e}^{\frac{a}{2 \nu} x} \sin \left(l_{1} x\right) \mathbb{1}_{\left[0, \frac{1}{2}\right]}(x)+\left(c_{2}^{+} \mathrm{e}^{\mathrm{i} k_{2}^{+} x}+c_{2}^{-} \mathrm{e}^{\mathrm{i} k_{2}^{-} x}\right) \cdot \mathbb{1}_{\left[\frac{1}{2}, 1\right]}(x) .
$$

The second condition in (6.3) is $c_{2}^{+} \mathrm{e}^{\mathrm{i} k_{2}^{+}}+c_{2}^{-} \mathrm{e}^{\mathrm{i} k_{2}^{-}}=0$, so that we get $c_{2}^{-}=-c_{2}^{+} \mathrm{e}^{\mathrm{i}\left(k_{2}^{+}-k_{2}^{-}\right)}=-c_{2}^{+} \mathrm{e}^{2 \mathrm{i} l_{2}}$. Setting $c_{2}=-2 \mathrm{i} c_{2}^{+}$and using (6.5) again, we get

$$
e_{m}=c_{1} \mathrm{e}^{\frac{a}{2 \nu} x} \sin \left(l_{1} x\right) \mathbb{1}_{\left[0, \frac{1}{2}\right]}(x)+c_{2} \mathrm{e}^{\mathrm{i} l_{2}} \mathrm{e}^{\frac{a}{2 \nu} x} \sin \left(l_{2}(1-x)\right) \mathbb{1}_{\left[\frac{1}{2}, 1\right]}(x) .
$$

Finally, the third condition in $(6.3)$ is $e_{m}\left(\left(\frac{1}{2}\right)^{-}\right)-e_{m}\left(\left(\frac{1}{2}\right)^{+}\right)=0$, leading to $c_{2}=c_{1} \frac{\sin \left(\frac{l_{1}}{2}\right)}{\sin \left(\frac{l_{2}}{2}\right)} \mathrm{e}^{-\mathrm{i} l_{2}}$, so that

$$
e_{m}(x)=\mathrm{e}^{\frac{a}{2 \nu} x}\left(\sin \left(l_{1} x\right) \mathbb{1}_{\left[0, \frac{1}{2}\right]}(x)+\frac{\sin \left(\frac{l_{1}}{2}\right)}{\sin \left(\frac{l_{2}}{2}\right)} \sin \left(l_{2}(1-x)\right) \mathbb{1}_{\left[\frac{1}{2}, 1\right]}(x)\right) .
$$

\subsection{Asymptotic expansion of the eigenvalues}

Now we look for the additional terms of the expansion of $l_{1}$ and $l_{2}$, for large $\lambda$. This expansion can be looked for as

$$
l_{2}=\pi m+\alpha+\frac{\beta}{m}+\frac{\gamma}{m^{2}}+o\left(\frac{1}{m^{2}}\right)
$$


Using (6.6) we get $l_{1}=\sqrt{l_{2}^{2}-\frac{K}{\nu}}$, and then $l_{1}=\pi m+\alpha^{\prime}+\frac{\beta^{\prime}}{m}+\frac{\gamma^{\prime}}{m^{2}}+o\left(\frac{1}{m^{2}}\right)$, with

$$
\alpha^{\prime}=\alpha, \quad \beta^{\prime}=\beta-\frac{K}{2 \nu \pi}, \quad \gamma^{\prime}=\gamma+\frac{\alpha K}{2 \nu \pi^{2}} .
$$

Then we have:

$$
\begin{aligned}
& \sin \left(\frac{l_{1}}{2}\right)=\sin \left(\frac{\pi m+\alpha}{2}\right) \cos \theta^{\prime}+\cos \left(\frac{\pi m+\alpha}{2}\right) \sin \theta^{\prime}+o\left(\frac{1}{m^{2}}\right) \\
& \cos \left(\frac{l_{1}}{2}\right)=\cos \left(\frac{\pi m+\alpha}{2}\right) \cos \theta^{\prime}-\sin \left(\frac{\pi m+\alpha}{2}\right) \sin \theta^{\prime}+o\left(\frac{1}{m^{2}}\right) \\
& \sin \left(\frac{l_{2}}{2}\right)=\sin \left(\frac{\pi m+\alpha}{2}\right) \cos \theta+\cos \left(\frac{\pi m+\alpha}{2}\right) \sin \theta+o\left(\frac{1}{m^{2}}\right) \\
& \cos \left(\frac{l_{2}}{2}\right)=\cos \left(\frac{\pi m+\alpha}{2}\right) \cos \theta-\sin \left(\frac{\pi m+\alpha}{2}\right) \sin \theta+o\left(\frac{1}{m^{2}}\right)
\end{aligned}
$$

with

$$
\begin{aligned}
& \cos \theta=\cos \left(\frac{\beta}{2 m}+\frac{\gamma}{2 m^{2}}\right)=1-\frac{\beta^{2}}{8 m^{2}}+o\left(\frac{1}{m^{2}}\right), \\
& \sin \theta=\sin \left(\frac{\beta}{2 m}+\frac{\gamma}{2 m^{2}}\right)=\frac{\beta}{2 m}+\frac{\gamma}{2 m^{2}}+o\left(\frac{1}{m^{2}}\right),
\end{aligned}
$$

and similar approximations for $\cos \theta^{\prime}$ and $\sin \theta^{\prime}$.

We then replace equations (6.9)-(6.10) into equation (6.7). The terms of order 1 in $m$ are:

$$
(\pi m+\alpha)\left(\sin \left(\frac{\pi m+\alpha}{2}\right) \cos \left(\frac{\pi m+\alpha}{2}\right)+\sin \left(\frac{\pi m+\alpha}{2}\right) \cos \left(\frac{\pi m+\alpha}{2}\right)\right)=0,
$$

leading to $\sin (\pi m+\alpha)=0$ and therefore $\alpha=0$. Consequently, (6.8) reads as:

$$
\beta^{\prime}=\beta-\frac{K}{2 \nu \pi}, \quad \gamma^{\prime}=\gamma
$$

The terms of order 0 in $m$ are then:

$$
\begin{aligned}
0 & =\pi m \cos ^{2}\left(\frac{\pi m}{2}\right) \frac{\beta^{\prime}}{2 m}-\pi m \sin ^{2}\left(\frac{\pi m}{2}\right) \frac{\beta}{2 m}-\pi m \sin ^{2}\left(\frac{\pi m}{2}\right) \frac{\beta^{\prime}}{2 m}+\pi m \cos ^{2}\left(\frac{\pi m}{2}\right) \frac{\beta}{2 m} \\
\Leftrightarrow \quad 0 & =(-1)^{m} \pi\left(\beta+\beta^{\prime}\right),
\end{aligned}
$$

leading to $\beta+\beta^{\prime}=0$ and then, thanks to $(6.11): \beta=\frac{K}{4 \nu \pi}$, and $\beta^{\prime}=-\frac{K}{4 \nu \pi}$.

Similarly, the terms of order -1 in $m$ lead to:

$$
0=\left(\sin ^{2}\left(\frac{\pi m}{2}\right)-\cos ^{2}\left(\frac{\pi m}{2}\right)\right)\left(\gamma^{\prime}+\gamma\right),
$$

so that $\gamma^{\prime}+\gamma=0$, and then, using (6.11): $\gamma=\gamma^{\prime}=0$.

We thus have the following expansions:

$$
l_{2, m}=\pi m+\frac{K}{4 \nu \pi m}+o\left(\frac{1}{m^{2}}\right), \quad l_{1, m}=\pi m-\frac{K}{4 \nu \pi m}+o\left(\frac{1}{m^{2}}\right) .
$$




\subsection{Proof of Theorem 2.4}

Let us now come back to our problem. Equation (6.1) can be written thanks to $A_{K}$ as

$$
\left\{\begin{aligned}
\partial_{t} w+A_{K}(w) & =0 \\
\left.w\right|_{x=0}=\left.w\right|_{x=1} & =0 \\
\left.w\right|_{t=0} & =w_{0}
\end{aligned}\right.
$$

To solve this equation, we decompose $w_{0}(x)$ on the basis $e_{m}: w_{0}(x)=\sum_{m \in \mathbb{Z}} w_{0}^{m} e_{m}(K ; x)$, and we get

$$
w(t, x)=\sum_{m \in \mathbb{Z}} w_{0}^{m} \mathrm{e}^{-\lambda_{m}(K) t} e_{m}(K ; x) .
$$

Similarly, the backward equation is

$$
\left\{\begin{aligned}
\partial_{t} \widetilde{w}+A_{-K^{\prime}}(\widetilde{w}) & =0 \\
\left.\widetilde{w}\right|_{x=0}=\left.\widetilde{w}\right|_{x=1} & =0 \\
\left.\widetilde{w}\right|_{t=0} & =\widetilde{w}_{0} .
\end{aligned}\right.
$$

And using the eigenvectors $e_{m}\left(-K^{\prime} ; x\right)$ of $A_{-K^{\prime}}$, if

$$
\widetilde{w}_{0}(x)=\sum_{m \in \mathbb{Z}} \widetilde{w}_{0}^{m} e_{m}\left(-K^{\prime} ; x\right)
$$

then $\widetilde{w}(t, x)=\sum_{m \in \mathbb{Z}} \widetilde{w}_{0}^{m} \mathrm{e}^{-\lambda_{m}\left(-K^{\prime}\right) t} e_{m}\left(-K^{\prime} ; x\right)$. So the Back and Forth Nudging problem $w(t=T)=\widetilde{w}(t=T)$ can be stated as follows: for each $n$, there exists a function $\widetilde{w}_{0}$ so that

$$
\mathrm{e}^{-\lambda_{n}(K) T} e_{n}(K ; x)=\sum_{m \in \mathbb{Z}} \widetilde{w}_{0}^{m} \mathrm{e}^{-\lambda_{m}\left(-K^{\prime}\right) T} e_{m}\left(-K^{\prime} ; x\right) .
$$

To improve readability, let us denote

$$
e_{m}(K ; x)=e_{m}(x), e_{m}\left(-K^{\prime} ; x\right)=\widetilde{e}_{m}(x), \quad \lambda_{n}(K)=\lambda_{n}, \lambda_{m}\left(-K^{\prime}\right)=\widetilde{\lambda}_{m}, \quad l_{i, m}=l_{i, m}(K), \widetilde{l}_{i, m}=l_{i, m}\left(-K^{\prime}\right) .
$$

Then the problem is to find $\widetilde{w}_{0}$ so that

$$
\mathrm{e}^{-\lambda_{n} T} e_{n}(x)=\sum_{m \in \mathbb{Z}} \widetilde{w}_{0}^{m} \mathrm{e}^{-\widetilde{\lambda}_{m} T} \widetilde{e}_{m}(x)
$$

As we will see later, the following basis $\left(f_{p}\right)$ is orthogonal to $\left(e_{m}\right)$ :

$$
f_{p}(x)=\mathrm{e}^{-\frac{a}{2 \nu} x}\left(\sin \left(l_{1} x\right) \mathbb{1}_{\left[0, \frac{1}{2}\right]}(x)+\frac{\sin \left(\frac{l_{1}}{2}\right)}{\sin \left(\frac{l_{2}}{2}\right)} \sin \left(l_{2}(1-x)\right) \mathbb{1}_{\left[\frac{1}{2}, 1\right]}(x)\right) .
$$

And similarly with $\left(\widetilde{e}_{m}\right)$ and $\left(\widetilde{f}_{p}\right)$.

To compute $\widetilde{w}_{p}^{0}$ we form the scalar product of equation (6.16) with $\widetilde{f}_{p}$, and we get:

$$
\widetilde{w}_{0}^{p}=\mathrm{e}^{\left(\widetilde{\lambda}_{p}-\lambda_{n}\right) T} \frac{\left(e_{n}(x) ; \widetilde{f}_{p}(x)\right)}{\left(\widetilde{e}_{p}(x) ; \widetilde{f}_{p}(x)\right)} .
$$


We are going to prove that this equation does not define a function, or even a distribution, because the coefficients $\widetilde{w}_{0}^{p}$ grow too fast with $p$ : indeed, we will show that the coefficients $\widetilde{w}_{0}^{p}$ grow as exponentials, therefore faster than any polynomial, and thus $\widetilde{w}$ is not defined in the distribution sense. To do so, we will provide asymptotic expansions of $\left(\widetilde{e}_{p}(x) ; \widetilde{f}_{p}(x)\right)$ and $\left(e_{n}(x) ; \widetilde{f}_{p}(x)\right)$ :

$$
\begin{aligned}
\left(\widetilde{e}_{p}(x) ; \widetilde{f}_{p}(x)\right)= & \int_{0}^{1} \mathrm{e}^{\frac{a}{2 \nu} x}\left(\sin \left(\widetilde{l}_{1, p} x\right) \mathbb{1}_{\left[0, \frac{1}{2}\right]}(x)+\frac{\sin \left(\frac{\widetilde{l}_{1, p}}{2}\right)}{\sin \left(\frac{\tilde{l}_{2, p}}{2}\right)} \sin \left(\widetilde{l}_{2, p}(1-x)\right) \mathbb{1}_{\left[\frac{1}{2}, 1\right]}(x)\right) \\
& \cdot \mathrm{e}^{-\frac{a}{2 \nu} x}\left(\sin \left(\widetilde{l}_{1, p} x\right) \mathbb{1}_{\left[0, \frac{1}{2}\right]}(x)+\frac{\sin \left(\frac{\tilde{l}_{1, p}}{2}\right)}{\sin \left(\frac{\tilde{l}_{2, p}}{2}\right)} \sin \left(\widetilde{l}_{2, p}(1-x)\right) \mathbb{1}_{\left[\frac{1}{2}, 1\right]}(x)\right) \mathrm{d} x \\
= & \frac{1}{4}\left(1+\left(\frac{\sin \left(\frac{\tilde{l}_{1, p}}{2}\right)}{\sin \left(\frac{\tilde{\tau}_{2, p}}{2}\right)}\right)^{2}\right)+\frac{1}{4}\left(-\sin \left(\widetilde{l}_{1, p}\right) / \widetilde{l}_{1, p}-\left(\frac{\sin \left(\frac{\tilde{l}_{1, p}}{2}\right)}{\sin \left(\frac{\tilde{l}_{2, p}}{2}\right)}\right)^{2} \sin \left(\widetilde{l}_{2, p}\right) / \widetilde{l}_{2, p}\right) .
\end{aligned}
$$

Using the asymptotic expansion (6.12) we get: $\frac{\sin \left(\widetilde{l}_{1, p}\right)}{\widetilde{l}_{1, p}}=\frac{\sin \left(\pi p+\frac{K^{\prime}}{4 \nu \pi p}+o\left(\frac{1}{p^{2}}\right)\right)}{\pi p+\frac{K^{\prime}}{4 \nu \pi p}+o\left(\frac{1}{p^{2}}\right)}= \pm\left(\frac{K^{\prime}}{4 \nu \pi^{2} p^{2}}+o\left(\frac{1}{p^{2}}\right)\right)$, and $\frac{\sin \left(\widetilde{l}_{2, p}\right)}{\widetilde{l}_{2, p}}=\frac{\sin \left(\pi p-\frac{K^{\prime}}{4 \nu \pi p}+o\left(\frac{1}{p^{2}}\right)\right)}{\pi p-\frac{K^{\prime}}{4 \nu \pi p}+o\left(\frac{1}{p^{2}}\right)}= \pm\left(-\frac{K^{\prime}}{4 \nu \pi^{2} p^{2}}+o\left(\frac{1}{p^{2}}\right)\right)$.

If $p$ is even, we have:

$\sin \left(\frac{\widetilde{l}_{1, p}}{2}\right)= \pm\left(\frac{K^{\prime}}{8 \nu \pi p}+o\left(\frac{1}{p^{2}}\right)\right), \quad \sin \left(\frac{\widetilde{l}_{2, p}}{2}\right)= \pm\left(-\frac{K^{\prime}}{8 \nu \pi p}+o\left(\frac{1}{p^{2}}\right)\right)$,

So that $\left(\frac{\sin \left(\frac{\tilde{l}_{1, p}}{2}\right)}{\sin \left(\frac{\tilde{l}_{2, p}}{2}\right)}\right)^{2}=1+o\left(\frac{1}{p}\right)$.

If $p$ is odd, then

$$
\sin \left(\frac{\widetilde{l}_{1, p}}{2}\right)= \pm\left(1+o\left(\frac{1}{p}\right)\right), \quad \sin \left(\frac{\widetilde{l}_{2, p}}{2}\right)= \pm\left(1+o\left(\frac{1}{p}\right)\right), \quad \text { and }\left(\frac{\sin \left(\frac{\tilde{l}_{1, p}}{2}\right)}{\sin \left(\frac{\widetilde{l}_{2, p}}{2}\right)}\right)^{2}=1+o\left(\frac{1}{p}\right) .
$$

Therefore we get

$$
\begin{aligned}
\left(\widetilde{e}_{p}(x) ; \widetilde{f}_{p}(x)\right) & =\frac{1}{4}\left(1+\left(\frac{\sin \left(\frac{\widetilde{l}_{1, p}}{2}\right)}{\sin \left(\frac{\widetilde{l}_{2, p}}{2}\right)}\right)^{2}\right)+\frac{1}{4}\left(-\sin \left(\widetilde{l}_{1, p}\right) / \widetilde{l}_{1, p}-\sin \left(\widetilde{l}_{2, p}\right) / \widetilde{l}_{2, p}\left(\frac{\sin \left(\frac{\widetilde{l}_{1, p}}{2}\right)}{\sin \left(\frac{\widetilde{l}_{2, p}}{2}\right)}\right)^{2}\right) \\
& =\frac{1}{2}+o\left(\frac{1}{p}\right) .
\end{aligned}
$$


Let us now compute $\left(e_{n}(x) ; \tilde{f}_{p}(x)\right)$ :

$$
\begin{aligned}
\left(e_{n}(x) ; \widetilde{f}_{p}(x)\right)= & \int_{0}^{1} \mathrm{e}^{\frac{a}{2 \nu} x}\left(\sin \left(l_{1, n} x\right) \mathbb{1}_{\left[0, \frac{1}{2}\right]}(x)+\frac{\sin \left(l_{1, n} / 2\right)}{\sin \left(l_{2, n} / 2\right)} \sin \left(l_{2, n}(1-x)\right) \mathbb{1}_{\left[\frac{1}{2}, 1\right]}(x)\right) \\
& \cdot \mathrm{e}^{-\frac{a}{2 \nu} x}\left(\sin \left(\widetilde{l}_{1, p} x\right) \mathbb{1}_{\left[0, \frac{1}{2}\right]}(x)+\frac{\sin \left(\widetilde{l}_{1, p} / 2\right)}{\sin \left(\widetilde{l}_{2, p} / 2\right)} \sin \left(\widetilde{l}_{2, p}(1-x)\right) \mathbb{1}_{\left[\frac{1}{2}, 1\right]}(x)\right) \mathrm{d} x \\
=\left(l_{1, n}^{2}-\widetilde{l}_{1, p}^{2}\right)^{-1}\left(l_{2, n}^{2}-\widetilde{l}_{2, p}^{2}\right)^{-1} & {\left[l_{1, n} l_{2, n}\left(-l_{2, n} \sin \left(\widetilde{l}_{1, p} / 2\right) \cos \left(l_{1, n} / 2\right)-l_{1, n} \cos \left(l_{2, n} / 2\right) \sin \left(\widetilde{l}_{1, p} / 2\right) \frac{\sin \left(l_{1, n} / 2\right)}{\sin \left(l_{2, n} / 2\right)}\right)\right.} \\
& +\widetilde{l}_{1, p} \widetilde{l}_{2, p}\left(-\widetilde{l}_{2, p} \sin \left(l_{1, n} / 2\right) \cos \left(\widetilde{l}_{1, p} / 2\right)-\widetilde{l}_{1, p} \cos \left(\widetilde{l}_{2, p} / 2\right) \sin \left(l_{1, n} / 2\right) \frac{\sin \left(\widetilde{l}_{1, p} / 2\right)}{\sin \left(\widetilde{l}_{2, p} / 2\right)}\right) \\
& +l_{1, n} \widetilde{l}_{2, p}\left(\widetilde{l}_{2, p} \sin \left(\widetilde{l}_{1, p} / 2\right) \cos \left(l_{1, n} / 2\right)+l_{1, n} \cos \left(\widetilde{l}_{2, p} / 2\right) \sin \left(l_{1, n} / 2\right) \frac{\sin \left(\widetilde{l}_{1, p} / 2\right)}{\sin \left(\widetilde{l}_{2, p} / 2\right)}\right) \\
& \left.+\widetilde{l}_{1, p} l_{2, n}\left(l_{2, n} \sin \left(l_{1, n} / 2\right) \cos \left(\widetilde{l}_{1, p} / 2\right)+\widetilde{l}_{1, p} \cos \left(l_{2, n} / 2\right) \sin \left(\widetilde{l}_{1, p} / 2\right) \frac{\sin \left(l_{1, n} / 2\right)}{\sin \left(l_{2, n} / 2\right)}\right)\right] .
\end{aligned}
$$

Now we use $l_{1, n}^{2}-l_{2, n}^{2}=-K / \nu$ and (6.7) to get:

$$
l_{1, n} l_{2, n}\left(-l_{2, n} \sin \left(\widetilde{l}_{1, p} / 2\right) \cos \left(l_{1, n} / 2\right)-l_{1, n} \cos \left(l_{2, n} / 2\right) \sin \left(\widetilde{l}_{1, p} / 2\right) \frac{\sin \left(l_{1, n} / 2\right)}{\sin \left(l_{2, n} / 2\right)}\right)=-\frac{K}{\nu} l_{1, n} \sin \left(\widetilde{l}_{1, p} / 2\right) \cos \left(l_{1, n} / 2\right) .
$$

Similarly, we get

$$
\widetilde{l}_{1, p} \widetilde{l}_{2, p}\left(-\widetilde{l}_{2, p} \sin \left(l_{1, n} / 2\right) \cos \left(\widetilde{l}_{1, p} / 2\right)-\widetilde{l}_{1, p} \cos \left(\widetilde{l}_{2, p} / 2\right) \sin \left(l_{1, n} / 2\right) \frac{\sin \left(\widetilde{l}_{1, p} / 2\right)}{\sin \left(\widetilde{l}_{2, p} / 2\right)}\right)=\frac{K^{\prime}}{\nu} \widetilde{l}_{1, p} \sin \left(l_{1, n} / 2\right) \cos \left(\widetilde{l}_{1, p} / 2\right) .
$$

And also using $l_{1, n}^{2}-l_{2, n}^{2}=-K / \nu, \widetilde{l}_{1, p}^{2}-\widetilde{l}_{2, p}^{2}=K^{\prime} / \nu$ and (6.7), the last two terms become:

$$
\begin{aligned}
& l_{1, n} \widetilde{l}_{2, p}\left(\widetilde{l}_{2, p} \sin \left(\widetilde{l}_{1, p} / 2\right) \cos \left(l_{1, n} / 2\right)+l_{1, n} \cos \left(\widetilde{l}_{2, p} / 2\right) \sin \left(l_{1, n} / 2\right) \frac{\sin \left(\widetilde{l}_{1, p} / 2\right)}{\sin \left(\widetilde{l}_{2, p} / 2\right)}\right) \\
& +\widetilde{l}_{1, p} l_{2, n}\left(l_{2, n} \sin \left(l_{1, n} / 2\right) \cos \left(\widetilde{l}_{1, p} / 2\right)+\widetilde{l}_{1, p} \cos \left(l_{2, n} / 2\right) \sin \left(\widetilde{l}_{1, p} / 2\right) \frac{\sin \left(l_{1, n} / 2\right)}{\sin \left(l_{2, n} / 2\right)}\right) \\
= & -l_{1, n}\left(K^{\prime} / \nu\right) \sin \left(\widetilde{l}_{1, p} / 2\right) \cos \left(l_{1, n} / 2\right)+\widetilde{l}_{1, p}(K / \nu) \sin \left(l_{1, n} / 2\right) \cos \left(\widetilde{l}_{1, p} / 2\right) .
\end{aligned}
$$

So that we get for $\left(e_{n}(x) ; \tilde{f}_{p}(x)\right)$ :

$$
\left(e_{n}(x) ; \widetilde{f}_{p}(x)\right)=\frac{K+K^{\prime}}{\nu}\left(l_{1, n}^{2}-\widetilde{l}_{1, p}^{2}\right)^{-1}\left(l_{2, n}^{2}-\widetilde{l}_{2, p}^{2}\right)^{-1}\left(\widetilde{l}_{1, p} \sin \left(l_{1, n} / 2\right) \cos \left(\widetilde{l}_{1, p} / 2\right)-l_{1, n} \sin \left(\widetilde{l}_{1, p} / 2\right) \cos \left(l_{1, n} / 2\right)\right) .
$$

In particular, for $K=-K^{\prime}$ and $n \neq p$, the vectors $\widetilde{f}_{p}$ are equal to $f_{p}$ and we get $\left(f_{p} ; e_{n}\right)=0$, and similarly $\left(\tilde{f}_{p} ; \widetilde{e}_{n}\right)=0$, as stated before.

We will now give an asymptotic expansion of (6.19). First we have, for fixed $n$ and large $p$ :

$$
\left(l_{1, n}^{2}-\widetilde{l}_{1, p}^{2}\right)^{-1}\left(l_{2, n}^{2}-\widetilde{l}_{2, p}^{2}\right)^{-1} \sim\left(l_{1, n}^{2}-\pi^{2} p^{2}\right)^{-1}\left(l_{2, n}^{2}-\pi^{2} p^{2}\right)^{-1}
$$


We will now give an expansion of $\widetilde{l}_{1, p} \sin \left(l_{1, n} / 2\right) \cos \left(\widetilde{l}_{1, p} / 2\right)-l_{1, n} \sin \left(\widetilde{l}_{1, p} / 2\right) \cos \left(l_{1, n} / 2\right)$. To do so, we use equations (6.9), (6.10) and (6.12):

$$
\begin{aligned}
& \widetilde{l}_{1, p} \sin \left(l_{1, n} / 2\right) \cos \left(\widetilde{l}_{1, p} / 2\right)-l_{1, n} \sin \left(\widetilde{l}_{1, p} / 2\right) \cos \left(l_{1, n} / 2\right) \\
= & \left(\pi p+\frac{K^{\prime}}{4 \pi \nu p}+o\left(\frac{1}{p^{2}}\right)\right) \sin \left(l_{1, n} / 2\right)\left(\cos (\pi p / 2)\left(1-\frac{\widetilde{\beta}^{\prime 2}}{8 p^{2}}+o\left(\frac{1}{p^{2}}\right)\right)-\sin (\pi p / 2)\left(\frac{\widetilde{\beta}}{2 p}+o\left(\frac{1}{p^{2}}\right)\right)\right) \\
& -l_{1, n} \cos \left(l_{1, n} / 2\right)\left(\sin (\pi p / 2)\left(1-\frac{\widetilde{\beta}^{\prime 2}}{8 p^{2}}+o\left(\frac{1}{p^{2}}\right)\right)+\cos (\pi p / 2)\left(\frac{\widetilde{\beta}^{\prime}}{2 p}+o\left(\frac{1}{p^{2}}\right)\right)\right),
\end{aligned}
$$

with $\widetilde{\beta}^{\prime}=\frac{K^{\prime}}{4 \nu \pi}$. Then for $p$ even we get:

$$
\begin{aligned}
& \widetilde{l}_{1, p} \sin \left(l_{1, n} / 2\right) \cos \left(\widetilde{l}_{1, p} / 2\right)-l_{1, n} \sin \left(\widetilde{l}_{1, p} / 2\right) \cos \left(l_{1, n} / 2\right) \\
& =(-1)^{p / 2}\left[\left(\pi p+\frac{K^{\prime}}{4 \pi \nu p}+o\left(\frac{1}{p^{2}}\right)\right) \sin \left(l_{1, n} / 2\right)\left(1-\frac{\widetilde{\beta}^{\prime 2}}{8 p^{2}}+o\left(\frac{1}{p^{2}}\right)\right)\right. \\
& \left.-l_{1, n} \cos \left(l_{1, n} / 2\right)\left(\frac{\widetilde{\beta}^{\prime}}{2 p}+o\left(\frac{1}{p^{2}}\right)\right)\right] .
\end{aligned}
$$

So that for $p$ large and even we have:

$$
\widetilde{l}_{1, p} \sin \left(l_{1, n} / 2\right) \cos \left(\widetilde{l}_{1, p} / 2\right)-l_{1, n} \sin \left(\widetilde{l}_{1, p} / 2\right) \cos \left(l_{1, n} / 2\right) \sim(-1)^{p / 2} \pi p \sin \left(l_{1, n} / 2\right),
$$

with $\sin \left(l_{1, n} / 2\right) \neq 0$. For $p$ odd, we get:

$$
\begin{aligned}
& \widetilde{l}_{1, p} \sin \left(l_{1, n} / 2\right) \cos \left(\widetilde{l}_{1, p} / 2\right)-l_{1, n} \sin \left(\widetilde{l}_{1, p} / 2\right) \cos \left(l_{1, n} / 2\right) \\
& =(-1)^{(p-1) / 2}\left[-\left(\pi p+\frac{K^{\prime}}{4 \pi \nu p}+o\left(\frac{1}{p^{2}}\right)\right) \sin \left(l_{1, n} / 2\right)\left(\frac{\widetilde{\beta}^{\prime}}{2 p}+o\left(\frac{1}{p^{2}}\right)\right)\right. \\
& \left.\quad-l_{1, n} \cos \left(l_{1, n} / 2\right)\left(1-\frac{\widetilde{\beta}^{\prime 2}}{8 p^{2}}+o\left(\frac{1}{p^{2}}\right)\right)\right] .
\end{aligned}
$$

So that for $p$ large and odd we have:

$$
\widetilde{l}_{1, p} \sin \left(l_{1, n} / 2\right) \cos \left(\widetilde{l}_{1, p} / 2\right)-l_{1, n} \sin \left(\widetilde{l}_{1, p} / 2\right) \cos \left(l_{1, n} / 2\right) \sim(-1)^{(p-1) / 2}\left[-\frac{K^{\prime}}{8 \nu} \sin \left(l_{1, n} / 2\right)-l_{1, n} \cos \left(l_{1, n} / 2\right)\right],
$$

which is a constant (possibly zero), up to the sign.

Equations $(6.17),(6.18),(6.20),(6.21)$ and $(6.22)$ show that the sequence $\left(\widetilde{w}_{0}^{p}\right)$ is not bounded by any polynomial in $\widetilde{\lambda}_{p}$, and therefore (6.15) does not define a distribution, so that the Back and Forth Nudging problem does not have a solution.

Acknowledgements. The authors are thankful to Prof. G. Lebeau (University of Nice Sophia-Antipolis) for his fruitful ideas and comments. This work has been partially supported by ANR JCJC07 and INSU-CNRS LEFE projects. 


\section{REFERENCES}

[1] D. Auroux and J. Blum, Back and forth nudging algorithm for data assimilation problems. C. R. Acad. Sci. Paris Sér. I 340 (2005) 873-878.

[2] D. Auroux and J. Blum, A nudging-based data assimilation method for oceanographic problems: the back and forth nudging (BFN) algorithm. Nonlin. Proc. Geophys. 15 (2008) 305-319.

[3] D. Auroux and S. Bonnabel, Symmetry-based observers for some water-tank problems. IEEE Trans. Automat. Contr. (2010) DOI: 10.1109/TAC.2010.2067291.

[4] H. Brezis, Analyse fonctionnelle : théorie et applications. Dunod, Paris (1999).

[5] R. Courant and D. Hilbert, Methods of Mathematical Physics II. Wiley-Interscience (1962).

[6] L.C. Evans, Partial Differential Equations. American Mathematical Society, Providence (1998).

[7] G. Evensen and P.J. van Leeuwen, An ensemble Kalman smoother for nonlinear dynamics. Mon. Weather Rev. 128 (1999) 1852-1867.

[8] B.-Z. Guo and W. Guo, The strong stabilization of a one-dimensional wave equation by non-collocated dynamic boundary feedback control. Automatica 45 (2009) 790-797.

[9] B.-Z. Guo and Z.-C. Shao, Stabilization of an abstract second order system with application to wave equations under non-collocated control and observations. Syst. Control Lett. 58 (2009) 334-341.

[10] J. Hoke and R.A. Anthes, The initialization of numerical models by a dynamic initialization technique. Mon. Weather Rev. 104 (1976) 1551-1556.

[11] R.E. Kalman, A new approach to linear filtering and prediction problems. Trans. ASME - J. Basic Eng. 82 (1960) $35-45$.

[12] E. Kalnay, Atmospheric modeling, data assimilation and predictability. Cambridge University Press (2003).

[13] M. Krstic, L. Magnis and R. Vazquez, Nonlinear control of the viscous burgers equation: Trajectory generation, tracking, and observer design. J. Dyn. Sys. Meas. Control 131 (2009) 1-8.

[14] F.-X. Le Dimet and O. Talagrand, Variational algorithms for analysis and assimilation of meteorological observations: theoretical aspects. Tellus 38A (1986) 97-110.

[15] D. Luenberger, Observers for multivariable systems. IEEE Trans. Automat. Contr. 11 (1966) $190-197$.

[16] Ph. Moireau, D. Chapelle and P. Le Tallec, Filtering for distributed mechanical systems using position measurements: perspectives in medical imaging. Inver. Probl. 25 (2009) 035010.

[17] K. Ramdani, M. Tucsnak and G. Weiss, Recovering the initial state of an infinite-dimensional system using observers. Automatica 46 (2010) 1616-1625.

[18] D.L. Russell, Controllability and stabilizability theory for linear partial differential equations: recent progress and open questions. SIAM Rev. 20 (1978) 639-739.

[19] A. Smyshlyaev and M. Krstic, Backstepping observers for a class of parabolic PDEs. Syst. Control Lett. 54 (2005) 613-625. 